\title{
Meso-scale modelling of compressive fracture in concrete with irregularly shaped
} aggregates

\author{
Sadjad Naderi, Wenlin Tu, Mingzhong Zhang* \\ Department of Civil, Environmental and Geomatic Engineering, University College London, \\ London, WC1E 6BT, UK
}

\begin{abstract}
This paper presents a meso-scale modelling framework to investigate the fracture process in concrete subjected to uniaxial and biaxial compression accounting for its mesostructural characteristics. 3D mesostructure of concrete consisting of coarse aggregates, mortar and interfacial transition zone between them was developed using an in-house code based on the Voronoi tessellation and splining method, which enables to generate the realistic-look aggregates with controllable structural features such as content, location, size and shape. Based on the generated 3D mesostructure, the concrete damage plasticity approach was employed to simulate the compressive fracture behaviour of concrete in terms of crack morphology and stress-strain response against the shape parameters of aggregate. Results indicate that the shape of aggregate has a negligible effect on compressive strength of concrete, which is highly associated with the random location and size distribution of aggregate. The aggregate irregularity has a significant influence on crack initiation and growth of concrete.
\end{abstract}

Keywords: Mesostructure; Irregular shape aggregate; Crack propagation; Damage evolution; Finite element analysis.

\section{Introduction}

Concrete is the most widely used construction material in the world. To date, tremendous efforts have been made to enhance the characterisation of concrete fracture using different experimental and analytical approaches [1]. However, it was found that the mechanical response is difficult to be faithfully captured during damage process in experiments, while the analytical methods may become very complex due to nonlinearity and randomness in a heterogeneous material like concrete [2-8]. The numerical approaches seem to be more effective to predict the fracture behaviour of concrete, among which the mesostructure-based models have been attracting more attention as the mesoscopic properties of concrete play an important role in fracture process in concrete [9-14]. These models make it possible to explicitly investigate the effects of structural features of concrete on fracture behaviour at the mesoscopic level, where concrete can be treated as a multiphase composite material consisting of coarse aggregates, mortar matrix and interfacial transition zone (ITZ) between them [15-17].

In order to accurately analyse damage mechanism, a mesostructure model is expected to provide

\footnotetext{
* Corresponding author. E-mail address: mingzhong.zhang@ucl.ac.uk (M. Zhang)
} 
reliable quantitative and qualitative data on fracture process, which can be characterised by both stress-strain response [18] and crack pattern properties [19-22]. The crack morphology does not only have a significant influence on mechanical strengths but also on transport properties such as permeability, diffusivity and absorptivity, which are strongly related to the long-term durability of concrete [1]. For instance, the crack development can facilitate the penetration of aggressive species (e.g. chlorides) into concrete, leading to deterioration of concrete and shortened service life of concrete structures. In recent years, numerous meso-scale models of concrete with the simplified geometries (e.g. 2D framework or using spherical-shaped aggregates) have been proposed, which might be able to predict the stress-strain response of concrete with an adequate precision but cannot properly simulate crack propagation in complex 3D mesostructures of concrete. The stress-strain data alone would be insufficient for the structural design of concrete and it is very important to provide the credible information on crack morphology. Therefore, all critical morphologic features should be considered to generate a model with a proper level of accuracy.

The model fidelity can be evaluated by comparing the properties of virtual and real mesostructures based on three experimental criteria related to coarse aggregates, which represent three levels of accuracy in hierarchical order: (1) Volume fraction: it is also known as parking density that represents the volume of coarse aggregates occupying the whole unit cell. The volume fraction of aggregate is typically between 30 to $50 \%$ based on the mix design suggested for normal strength concrete [18]. In terms of aggregate size, this is the lowest level of accuracy required to be considered in a model. Most of the previous models were able to adjust the volume fraction of aggregates [ㅇ, 18, 23]. (2) Size distribution: it is a list of values or a mathematical function that indicates the relative amount of aggregate present according to size [18]. A model adjusted based on this criterion meets the acceptable standard of validity regarding aggregate size. The previous studies showed that it was feasible to fulfil it in a model $[9, \underline{18}, \underline{23}, \underline{24}]$. (3) Shape: the highest accuracy would be reached if both the size distribution and shape of aggregates could be configured in reference to the real data. Although, the complications start here in terms of measurement and simulation techniques due to the irregular nature of aggregates. Several shape parameters may be required to quantitively describe the morphology, while even the accuracy of measurement methods would be disputable. The shape parameters obtained from the empirical data are the only way to validate a model. Regarding simulation, it is not easy to model irregular aggregates in random media with the desired shape properties and size distribution. In the following, the key issues will be more discussed on the simulation of aggregates with the realistic morphology.

In general, two main approaches are used to simulate a mesostructure based on computational geometric algorithms including image-dependent and image-independent methods [25]. An imagedependent approach can produce a realistic model by scanning an actual sample. However, it is a 
time-consuming and computationally expensive method and is highly dependent on image resolution [26-32]. These specifications limit the shape variations and number of models, which can be possibly produced. Consequently, it causes some difficulties in a typical statistical analysis, which is an essential part of the study of such random system. In contrast, the image-independent method is known as a computational cost-effective methodology with more producibility and less restriction of real samples [33]. Nevertheless, the actual aggregate shape was disregarded by using the simplified geometry in many previous models $[\underline{2}, \underline{15}, \underline{34-39}]$.

To address the limitations mentioned, this study aims to develop a novel modelling framework with a high level of accuracy and low computational cost to investigate the effect of 3D mesostructure of concrete on crack morphology. The framework is based on the previous work [25] on the development of the image-independent simulation tools to generate the 3D particulate mesostructure model using the combination of Voronoi tessellation method and splining technique. It could create a realistic-looking geometry model with controllable structural parameters including shape, size and distribution of particles. This integrated model has two remarkable capabilities: (1) The model programmability allows designing a methodology that integrates the undiscovered aspects of fracture analysis affected by the irregular-shape of aggregates. (2) The controllability of the model enables to systematically adjust the overall and local shape of the aggregates in regard to the shape parameters including sphericity, elongation and roundness, which can be used to describe irregularity [25]. Thus, the mechanical response can be quantitatively characterised against the shape parameters. The triangulated surface representation of the aggregate model eases the measurement of these parameters and the simulation process of ITZ around each aggregate. The geometric model generated with the configuration designed can be applied to the continuum-based nonlinear finite element (FE) analysis to simulate the concrete fracture. Among different material models, concrete damage plasticity (CDP) that has been successfully developed, used and tested in many case studies [40-46] is adopted to predict the post-peak softening and crack pattern through mortar and ITZ in this study. Its combination with the mesostructure model proposed can precisely simulate two main failure modes including tensile cracking and compressive crushing.

In this paper, the fracture process in concrete under uniaxial and biaxial compression is investigated accounting for its mesostructural characteristics. Firstly, the 3D mesostructure of concrete composed of coarse aggregates, ITZ and mortar are generated by the in-house package developed in MATLAB considering the content, random location, size distribution and shape of aggregate. The size distribution of aggregate is adjusted based on the sieve analysis obtained from the experiments, while the shape properties are set up using a systematic strategy to gradually change the irregularity level. The spherical and polyhedron shaped aggregates involved in this study highlight the effect of geometric simplifications. The method to generate spherical aggregates follows the 
conventional algorithm, as presented in the literature $[\underline{2}, \underline{15}, \underline{34}, \underline{35}]$. The simulated aggregates are compared with two of the most common aggregates used for concrete production, i.e. natural gravels and crushed rocks [47]. Because of their structural characteristics, they cover a wide range of shape variations required for a comprehensive analysis targeted here. Natural gravels represent rounded aggregates with a quasi-spherical geometry and crushed rocks stand for rough aggregates with angular, elongated or flaky structure. Afterwards, based on the generated 3D mesostructure of concrete and CDP material model, the FE simulations are carried out using ABAQUS/Explicit to explore the fracture process in concrete under compression in terms of stress-strain response and crack morphology. Finally, the effects of shape parameters of aggregate on concrete fracture behaviour are estimated and discussed in detail.

\section{3D mesostructure of concrete}

The principle of the 3D mesostructure model is tied to the seed points used for the Voronoi tessellation $[25, \underline{48}]$. The seed points dictate the discretisation mode of the domain and control the structural attribute of the Voronoi cells. In general, the point distribution pattern and the splining process are employed to adjust the aggregate shape. The details can be referred to the previous work [25]. In the following, the shape and size configurations will be presented for the splined Voronoi cells, which are used to simulate the aggregates in a cubic representative volume element (RVE). The side length of the RVE is set to $50 \mathrm{~mm}$ in order to meet the criterion that the RVE size should be at least three to five times greater than the maximum size of the aggregate to achieve a reportative volume [9].

\subsection{Aggregate shape configurations}

The seed points are systematically distributed to control the irregularity through the mesostructure. First, a uniform non-random distribution of points is used to yield a model with regularshaped/distributed cells as a reference to define the irregularity level. Fig. 1a shows an example of this regular system with honeycomb-shaped Voronoi cells, which have body-centred cubic (BCC) structure. The irregularity is then induced by the perturbation of points' coordinates. Each point is displaced by the vector $\vec{r}$ with random length and direction, from where the seed points originally located in the reference model. By increasing the maximum length of the displacement vector $\vec{r}$, the seed points become more disordered. Consequently, the Voronoi cells get more distorted and their regular shape becomes more irregular. The variable $r$ represents the irregularity level, which can be adjusted for each model. Three Voronoi tessellation models shown in Fig. 1 schematically exemplify different irregularity levels, which will be used for the mesostructure generation, corresponding to the $r$ values of $0,0.05$ and 0.2 unit (the side length of the squares is 1 unit). As seen, the irregular attribute is associated with several factors including the random location, size, shape of the cells in the RVE whereas the cell structure is irregular itself.

The splining technique is employed to transform the Voronoi cell structure to a geometry, which 
looks more like the real aggregates. Mainly, this technique regularises the sharp edges and corners of the polyhedral cells into a more rounded shape. In other words, it converts the flat faces to curved ones and reduces the high angularity. Fig. 2 simply illustrates how the original Voronoi cell changes to the splined cells. It should be mentioned that the splining process is based on subdividing surfaces and it can be done in multi-subdivision iterations. A smoother surface is created after each iteration level. In this study, two iteration levels are applied. A more accurate description of the transformations created by the seed points distribution and the splining process will be given later using the shape parameters while comparing different types of aggregate shape considered here.

For a complete study of the shape effect, aggregates are simulated by three types of geometry including spheres, polyhedral Voronoi cells and splined-Voronoi cells as exemplified in both Table 1 by the single particles and Fig. 3 by the whole set of aggregates. The models are labelled based on the geometric type, the irregularity and the splining iteration level as given in Table 1. The main concepts of designing these systems are described as follows and summarised in Table 2. The models $\boldsymbol{A}$ and $\boldsymbol{D}$ as the simplified versions of the aggregate model have been traditionally and repeatedly used in the literature, e.g. [49-55]. The analyse of such models in the framework proposed provides insight on their efficiency against $\boldsymbol{G}$. Also, in a physical sense, these two models are likely comparable to natural gravels and crushed rocks due to the geometric similarities. For visual comparison, the samples of the real aggregates are shown in Fig.4. Although the low-irregularity characteristic of the models $\boldsymbol{B}, \boldsymbol{C}, \boldsymbol{E}$ and $\boldsymbol{F}$ is inconsistent with the random nature of concrete, these models simulate the gradual shape transformations by increasing irregularity for each geometric type. Therefore, they allow general understanding the influence of irregularity by comparing the results between the models with the same geometric type. Due to the reason stated above, only the models $\boldsymbol{D}$ and $\boldsymbol{G}$ among the others represent the mesostructures with the polyhedral Voronoi and splined-Voronoi cells, respectively. They can be compared with the model $\boldsymbol{A}$ and each other to show the effect of geometric type and the splining process accordingly.

So far, the overall shape characteristics have been presented and their relationship with the analysis methodology has been explained. Now, thanks to the measurability and the controllability of the models, the structural characteristics can be described and discussed by the shape parameters including sphericity, elongation ratio and roundness. Sphericity and elongation ratio are used to describe the global shape while the roundness is a descriptor of the local surface shape. Sphericity and elongation ratio can be defined as the deviation of an irregular-shaped aggregate from the regular perfect sphere. Here, the following equations [25] are used to calculate these parameters by the aid of the triangle elements that make the cell surface: 
Sphericity $=\frac{\text { Surface Area of Equivalent Sphere }}{\text { Surface Area of Particle }}=\frac{4 \pi\left(\frac{3 V}{4 \pi}\right)^{2 / 3}}{S}$

Elongation ratio $=\frac{L_{\min }}{L_{\max }}$

Roundness $=\frac{\sum_{i=1}^{m}\left|\hat{r}_{i} \cdot \hat{n}_{i}\right| \triangle S_{i}}{S}$

where $V$ and $S$ are the volume and surface area, respectively, $L$ is the distance between the centre of the cell and a node on the surface, $\hat{r}_{i} . \hat{n}_{i}$ denotes the angle between a vector from cell centroid to centre of the $i$-th triangle element and the corresponding normal vector of the element, $\triangle S_{i}$ is the area of the element, and $m$ represents the total number of elements for the cell.

The values of the above-mentioned three shape parameters range from zero to one. A module to measure the parameters is incorporated into the MATLAB code for mesostructure generation. Therefore, the geometry models generated are accompanied with the data, which includes the values of the shape parameters for all particles. Regarding this, a histogram can be used to show the shape distribution. However, it should be noted that 30 random samples are generated per model set to yield reliable results in the statistical analysis. To obtain a representative histogram for each set, first, the entire range of values should be divided into the same series of intervals (or bins) for all samples in a set. Second, it is counted how many values fall into each interval per sample. Third, a mean value per interval is calculated by averaging the data in the corresponding interval for all models. Fig. 5 illustrates the representative histograms of the shape parameters with a normal probability density function (PDF) fitted from the distribution specified for all sets except the model $\boldsymbol{A}$. The standard deviation for each bin is less than 0.1 for all plots, which proves that number of the models is enough in terms of shape. The sufficiency of the number of samples regarding the mechanical properties will be discussed later. The PDF curves help to better understand the shape transformation. The arrows are used as a guide for the eyes in a comparative view between sets of polyhedral Voronoi and splinedVoronoi cells with an equal irregularity level.

By comparing the models $\boldsymbol{D}$ and $\boldsymbol{G}$ with the real aggregates, it can be concluded that the simulated ranges of shape parameters are generally consistent with the experimental data. The sphericity values of natural gravels and crushed rocks are within the range of 0.6 to 0.9 [56-58]. This is almost equal to the range reported in Fig. 5 for the models $\boldsymbol{D}(0.6$ to 0.85$)$ and $\boldsymbol{G}(0.65$ to 0.95$)$. Similarly, the roundness ranges are compatible with the experimental values, which are from 0.6 to 0.8 [58]. The elongation ratio relatively varies in a wider range in both simulation (0.2 to 0.6$)$ and experiments $(0.3$ to 0.7$)$ [56]. By comparing the models $(\boldsymbol{E}, \boldsymbol{F}, \boldsymbol{G})$ and $(\boldsymbol{B}, \boldsymbol{C}, \boldsymbol{D})$ separately, the effect of irregularity on the shape distribution can be highlighted. As more cells with different shapes are created in RVEs by 
increasing $r$, the values of the shape indices are distributed more broadly. By comparison of the models $(\boldsymbol{D}, \boldsymbol{G}),(\boldsymbol{C}, \boldsymbol{F})$ and $(\boldsymbol{B}, \boldsymbol{E})$ for each shape parameter, it can be found that the sphericity and the roundness values increase about $10 \%$ after splining process where, conversely, the elongation ratio slightly decreases.

In this section, the models were categorised into different sets and introduced based on the aggregate shape configurations. To complete the information about the structural characteristics, the size distribution of the aggregate models will be presented hereafter.

\subsection{Aggregate size distribution}

The sieve analysis is conducted to measure the mass fraction of particles within each size range to determine the aggregate size distribution (ASD), which can be described by means of the grading curves such as the Fuller curve [25]. The aggregate grading designed according to the Fuller curve leads to an optimum concrete mixture, which can be expressed as: [1]

$$
P(d)=100\left(\frac{d}{d_{\max }}\right)^{n}
$$

where $P(d)$ is the cumulative percentage passing a sieve with aperture diameter $d, d_{\max }$ is the maximum size of aggregate particles, and $n$ is the exponent of the equation (commonly $\mathrm{n}=0.5$ ).

To facilitate the implementation of the gradation curve in a numerical model, it can be divided into segments, where the amount of aggregates $\mathrm{V}_{\text {agg }}$ within each grading segment $\left[d_{i}, d_{i+1}\right]$ can be determined by:

$$
V_{a g g}\left[d_{i}, d_{i+1}\right]=\frac{P\left(d_{i+1}\right)-P\left(d_{i}\right)}{P\left(d_{\max }\right)-P\left(d_{\min }\right)} \times P_{a g g} \times V_{\text {total }}
$$

where $d_{\max }$ and $d_{\min }$ are the maximum and minimum aggregate size, respectively, $P_{a g g}$ denotes the volume fraction of aggregates, and $V_{\text {total }}$ is the total volume of concrete specimen.

A typical ASD with four sieve groups [59] as given in Table 3 is used, which categorises the coarse aggregates into three size ranges including small (2.36 to $4.75 \mathrm{~mm}$ ), medium (4.75 to $9.5 \mathrm{~mm}$ ) and large ( 9.5 to $12.7 \mathrm{~mm}$ ). To adjust the cells, the calibration process is performed on the models. The algorithm proposed for calibration was presented in [25]. It is assumed that the aggregates occupy $30 \%$ of the total concrete volume. In Fig. 3, the size segmentations are accordingly specified by different colours of the aggregate models. Similar to the shape distribution, the mean values of the aggregate probability density in each size range are plotted in Fig. 6 for all samples in each set.

\subsection{ITZ and mortar}

ITZ is usually considered as the weak region between aggregate and mortar in concrete due to the relatively high water-to-cement ratio and porosity in this region. ITZ is generally modelled as a thin layer around each aggregate with a typical thickness ranging from 0.01 to $0.05 \mathrm{~mm}$ [48], which is extremely low compared to the size of aggregate. Such size difference causes difficulties in meshing. 
If the coarse elements (consistent with aggregate size) are used, the mesh quality might become very poor. If the small elements (consistent with ITZ thickness) are used, the number of elements might drastically increase and consequently computational cost. In the previous studies, the thickness was sometimes exaggerated $(\sim 0.1$ to $1 \mathrm{~mm})$ due to these issues [ $\underline{48}, \underline{60-63}]$. Nevertheless, in this study, the approach to model ITZ allows to set thickness as $0.05 \mathrm{~mm}$ while the mesh quality is kept acceptable. The ITZ layer is created by the aid of an offset surface generated upon the aggregate surface. The region between the aggregate and the corresponding offset surface is meshed by the solid elements and in this way, the ITZ is constructed. The generation of the offset surface is performed based on the scaling technique [25] and coded in MATLAB. The modelling procedure consists of two main steps: (1) the triangulated representation of the aggregate surface is duplicated by reproducing the matrix of vertices (or nodes); (2) the duplicated geometry is expanded with reference to the centroid position whilst the distances between the original and the duplicated nodes are equal to the ITZ thickness. It is important to be noted that the aggregates do not overlap with each other after expansion, as the minimum distance between two particles was set greater than double thickness of ITZ earlier in the size adjustment process of the aggregates.

Similarly, the mortar is modelled by meshing the region created through the offset surfaces mentioned above and the exterior surfaces of the RVE. The modelling experience proved that the simulation strategy proposed was computationally reasonable and compatible with this framework. Fig.7 shows an example of the generated 3D mesostructure of concrete composed of coarse aggregates, mortar matrix and ITZs between them. The cross-sectioned models depict the composition of the mortar matrix, aggregate particles and the ITZ layers in the RVE.

\section{Finite element modelling}

\subsection{Material model}

For normal concrete, the tensile and compressive strengths of the aggregates are much higher than those of mortar and ITZs [40]. Thus, the aggregate can be assumed to be elastic without damage allowed whereas the nonlinear behaviour and damage occur prior to concrete failure in the mortar and the ITZs. The CDP model, available in the ABAQUS material library, is used to simulate damage in both mortar and ITZs. The theory behind this continuum plastic model has been documented in ABAQUS [64] and described many times in the literature [ㄷ]. Therefore, only the key points are presented here.

For both mortar and ITZs, the stress-strain relations are simplified to be linear before reaching the yield point in compression and the ultimate strength in tension. The compressive strain hardening and softening curves can be approximated by different constitutive relations $[\underline{18}, \underline{48}, \underline{66}]$. The following equations are adopted to define the hardening and post-peak softening curves according to GB50010 


$$
\frac{\sigma_{c}}{f_{c}}= \begin{cases}\alpha_{a} \frac{\varepsilon_{c}}{\varepsilon_{c u}}+\left(3-2 \alpha_{a}\right)\left(\frac{\varepsilon_{c}}{\varepsilon_{c u}}\right)^{2}+\left(\alpha_{a}-2\right)\left(\frac{\varepsilon_{c}}{\varepsilon_{c u}}\right)^{3} & \text { Hardening } \\ \frac{\varepsilon_{c}}{\varepsilon_{c u}} & \text { Softening } \\ \alpha_{d}\left(\frac{\varepsilon_{c}}{\varepsilon_{c u}}-1\right)^{2}+\frac{\varepsilon_{c}}{\varepsilon_{c u}} & \end{cases}
$$

where $\sigma_{c}$ and $\varepsilon_{c}$ are the compressive stress and strain, respectively, $\varepsilon_{c u}$ is the ultimate compressive strain corresponding to its strength $f_{c}$, and $\alpha_{a}$ and $\alpha_{d}$ are the coefficients that can be calculated by:

$$
\left\{\begin{array}{l}
\alpha_{a}=2.4-0.0125 f_{c} \\
\alpha_{d}=0.157 f_{c}^{0.785}-0.905
\end{array}\right.
$$

The tensile softening behaviour is approximated by the linear fracture energy-based criterion to avoid unreasonable mesh sensitivity results [66]. Regarding this, the keyword *CONCRETE TENSION STEFFEINING, TYPE=DISPLACEMENT is used in ABAQUS. Regarding the input parameters, the mechanical properties have been variously reported for mortar, ITZ and aggregates. As this research does not focus on the specific concrete, the acceptable values are selected as the inputs for the model based on the previous studies $[\underline{15}, \underline{18}, \underline{47}, \underline{68}]$ from the point of view of reliability and availability. The main material parameters used in the FE simulations are listed in Table 4. Moreover, five other parameters should be identified including the dilation angle, the plastic potential eccentricity, the ratio of the compressive strengths under biaxial and uniaxial loading, the ratio of second stress invariant on the tensile meridian to that on the compressive meridian, and the viscosity parameter. Their corresponding values $35^{\circ}, 0.1,1.16,0.667$ and 0.0005 are used for both mortar and ITZs in this study $[\underline{39}, \underline{69}]$.

The damage indices of DAMAGEC for compression, DAMAGET for tensile and SDEG as stiffness degradation variables are utilised to approximate the cracks induced. They evolve monotonically from 0 (undamaged) to 1 (fully damaged) to indicate the damage level. In contrast with the discrete crack models, the continuum mechanics-based approach like the CDP model is not capable to provide accurate data such as crack width. However, it can effectively simulate the crack pattern that is the main focus of this study.

\subsection{Loads and boundary conditions}

The displacement control method is used to apply the quasi-static load. For uniaxial loading, the vertical displacement is prescribed on one face of the RVE while its opposite face is fixed. For biaxial compression, the same approach is used but for two faces. In order to cover a wide enough range of the stress-strain response in the softening behaviour, the simulation is terminated at a maximum loading displacement $d$ of $0.25 \mathrm{~mm}$. It is adopted with a long enough loading time of $0.02 \mathrm{~s}$ to minimise the potential dynamic effect, which will be discussed in the following section.

\subsection{Model Reliability}

To evaluate the reliability of the model, the influences of parameters such as element size, time step 
and sample number on simulation results are studied. The sensitivity analysis is carried out to obtain the most suitable values considering accuracy and computational efficiency. In addition, the simulation results are compared with literature data to support the validation.

\subsubsection{Element size (mesh density)}

The choice of mesh size has a crucial influence on the simulation of crack growth. Very small mesh size would lead to unnecessary computational overhead, whereas the opposite may yield inaccurate data [18]. Hence, the ideal mesh size should be fine enough and consistent with such complex geometry to precisely capture the cracking and stress-strain behaviour with a reasonable computational cost. To find an adequate mesh size, a model of the set $\boldsymbol{A}$ is meshed with four sizes. In the non-uniform mesh generation, the maximum element characteristic length $\mathrm{L}_{\mathrm{e}}$ is set to $0.8,1,1.2$ and $1.4 \mathrm{~mm}$. The corresponding number of elements is around 2.8, 2.1, 1.5 and 1 million, respectively. The simulated stress-strain curves for each element size for uniaxial loading are illustrated in Fig. 8. The stress is calculated as the sum of the nodal reaction forces on the loaded face divided by the initial face's area $\left(50 \times 50 \mathrm{~mm}^{2}\right)$. The macroscopic engineering strain is obtained from the average nodal displacement divided by the initial side length of the RVE $(50 \mathrm{~mm})$. It can be observed that the mesh dependence of the stress-strain curves is negligible for the models when $\mathrm{L}_{\mathrm{e}}$ is less than $1.2 \mathrm{~mm}$. Considering the balance between the accuracy and computational efficiency, $\mathrm{L}_{\mathrm{e}}$ is therefore adjusted to $1 \mathrm{~mm}$ in the mesh setting for all the models.

\subsubsection{Loading time}

Loading time is an important parameter in an explicit quasi-static solution, which is employed to overcome the convergence difficulties related to fracture simulation. Theoretically, the loading time should be long enough to avoid any dynamic effect, although the computational cost rises inevitably by increasing time. Thus, it is desirable to set the time as less as possible. For this, the model sensitivity of this parameter is investigated on a model of the set $\boldsymbol{A}$ with three different loading times of $0.01,0.02$ and 0.025 s. Fig. 8 demonstrates the simulated stress-strain response for uniaxial compression. It can be depicted from the curves that the loading time dependence can be almost negligible for times longer than $0.02 \mathrm{~s}$. Consequently, the loading time is adjusted to $0.02 \mathrm{~s}$ for all sets. As an assessment for the influence of the dynamic effect [70], the ratio of the total kinetics to the total internal energy was found to be always below 5\% during the simulation time for all models.

\subsubsection{Sample number}

To determine the sample number, the results of 30 random models of the set $\boldsymbol{A}$ are investigated. Fig. 9 shows the stress-strain response of concrete under uniaxial compression. The corresponding mean curve and the standard deviation of stress at different loading steps are also plotted in the figure. To better describe the stress-strain curve, three characteristic points, i.e. I, II and III, are identified based on the results, which specify the yield stress (or the end of the elastic region), the peak stress (or the 
material strength) and the critical stress/strain at the maximum standard deviation $(\varepsilon=0.002)$. As seen in Fig. 9, the deviation of the results is insignificant (less than $0.2 \mathrm{MPa}$ ) in the elastic (before Point I) and hardening stages (between Points I and II), while the value drastically increases from about Point II to the maximum at Point III. Therefore, the influence of sample number on the values of stress and standard variation at Points II and III as the critical parameters are examined. For this, the means of these parameters are calculated for all possible combinations of $n$ samples $(n \leq 30)$. The averages of the mean values are plotted in Fig. 10. Smaller fluctuations are observed for the sample numbers greater than 15 while the parameters tend to be constant, which indicates that 15 samples are likely large enough to get a statistically representative model. Nevertheless, 30 realisations per model set are used in this study to guarantee convergence.

\subsubsection{Comparison with other studies}

For comparison, the stress-strain curves of concrete obtained from experiments and numerical simulations that are presented in $[\underline{71}, \underline{72}]$ are plotted together with the simulated results of this study in the subplot of Fig. 9. The discrepancy of the curves can be probably explained by the difference of sample preparation, concrete features (e.g. void or aggregate content), etc. It is worth noting that this study does not aim to generalise the results but to gain insight and suggest hypothesis and directions for concrete design.

\section{Simulation results and discussion}

Distinct failure processes and deformation patterns in concrete can be recognised in relation to its mesostructural characteristics. To specify this, first, the main crack characteristics will be demonstrated. Then, the evolution of the cracks and their final morphology will be presented, which can be mostly observed in each set. The stress-strain response will be compared among the models based on the concepts, which were described in Section 2.1 and summarised in Table 2. Also, the dissipated energy and the critical values of stress at the characteristic points such as Points II and III (Fig. 9) will be analysed to estimate the effects of shape parameters.

\subsection{Crack mechanisms}

The fracture process in concrete can be revealed by the distribution of the displacement and damage fields. As an example, Fig. 11a shows $U_{y}$ and SDEG contour maps, which represent the displacement in loading direction and the compression-tension damage under uniaxial compression respectively for the model $\boldsymbol{G}$. Herein, it is assumed that the elements with SDEG values greater than 0.9 are regarded as the crack. The assumption can be acceptable since the damage generally diffuses in a limited distance through the intact phase for such brittle materials. The mortar discontinuity induced by the cracks is detectable by the displacement field as well. The fracture patterns observed among the models mainly look similar to the real samples tested under compression with a high frictional constraint condition [36]. As shown in Fig. 11b, the pattern can be described by the oblique cracks, 
indicating a triangular morphology. The final cracks mostly break a specimen into some pyramidal/triangular fragments, the height and bottom length of which are smaller than the initial specimen size. The triangle-like structure is characterised by the crack thickness $\delta$ and the so-called propagation angle $\theta$. Hereafter, these parameters will be qualitatively discussed through analysing the crack morphology. The occurrence of such crack branching can be attributed to the stress distribution regime, which dictates the fracture behaviour. The stress distribution itself is affected by the random mesostructural characteristics of the aggregates and their interactions with each other. The local stress level is mainly intensified around the aggregates, where the ITZs exist as the weakest material and in the small regions among them. Thus, the crack tends to initiate and propagate along the aggregate surfaces and the narrow paths in the mortar. The potential points for the damage initiation located in these regions are called nucleation points. Thus, the geometric characteristics of the aggregates have a significant effect on the nucleation and coalescence behaviour of the cracks.

According to the above description, the differences between the models are discussed by the aid of the representative samples. Figs. 12-14 illustrate the damage evolution under uniaxial compression at different stages of crack development using three visualisation approaches for the models $\boldsymbol{A}, \boldsymbol{D}$ and $\boldsymbol{G}$. The load is applied in X-direction. The growth of crack is captured after about Point III (indicated in Fig. 9) when the damage is clearly visible. In the rows (a), the crack is displayed by removing the relevant elements while the displacements in the loading direction and perpendicular to it are scaled by 30 and 10, respectively, for better visualisation. In the rows (b), the crack cluster is shown to demonstrate the damage propagation among the aggregates in 3D. In the rows (c), the cross-sectional view provides a better picture of crack branching in respect to the aggregates. In comparison of different sets, the distances among the aggregates would have same effects on cracking in all models but the number of nucleation points in $\boldsymbol{D}$ and $\boldsymbol{G}$ (Figs. 13 and 14) is higher than that in $\boldsymbol{A}$ (Fig. 12) due to the small local surface area, sharp edges and corners existed on the aggregate surface. It increases the formation probability of preliminary cracks in more locations and the propagation possibility in different directions. As seen in the row (c) of the figures, for the model $\boldsymbol{A}$ at $\varepsilon_{1}$, the crack nucleates between aggerates with less distance while for the models $\boldsymbol{D}$ and $\boldsymbol{G}$, it also starts around the sharp vertices. However, it seems that the model $\boldsymbol{G}$ is more realistic since neither it is perfectly rounded like a sphere, nor very angular like a polyhedral geometry. Thus, the number and locations of the nucleation points should be more consistent with the actual samples. At $\varepsilon_{4}$ when the final crack network is formed, it can be observed that the parameter $\theta$ is more non-uniform for the irregularly shaped aggregates. In other words, the energy is locally dissipated in more various sub-regions for these types of aggregates, whilst the local damage concentration is higher for the model $\boldsymbol{A}$. As the total amounts of the dissipation energy do not differ significantly between these model sets, it can be interpreted that the irregular aggregates cause the cracks to percolate through a greater area but with 
lower $\delta$. To further highlight, to what extent, the irregularity level might affect the fracture, the material discontinuity is visualised in Fig. 15 by a combination of the displacement distribution field and cracks for all model sets with the cross-sectional views. With the increase of irregularity level from $\boldsymbol{B}$ to $\boldsymbol{D}$ and $\boldsymbol{E}$ to $\boldsymbol{G}$, the thickness $\delta$ gets smaller and the non-uniformity of angle $\theta$ becomes greater. By comparison of all sets, it can be concluded that more irregularity causes the specimens to fragment to pieces with a relatively smaller size. Therefore, the compressive fragmentation mode would be dominated by the aggregates shape.

Under biaxial loading, the failure mode is more complicated, but some structural effects become more significant. Figs. 16-18 show the crack evolution under biaxial compression in X- and Ydirections. The method of visualisation is similar to the uniaxial case but in the rows (c), three crosssectional planes are displayed to illustrate the crack propagation in the loading directions and perpendicular to them. The pyramidal morphology of cracks detected in the uniaxial mode transforms to a rhomboid-like geometry, which is more obvious in Fig. 17a and b. The oblique layers of the cracks are initially formed and then while they are coalescing, $\delta$ of them are getting larger. The propagation angle $\theta$ is more recognisable on the planes perpendicular to the loaded faces. The damage mechanism is activated in two directions and so, the crack morphology would be more influenced by the shape of aggregates in comparison with the models under uniaxial loading. To estimate this, the volume fraction of the crack clusters is measured based on the elemental volume of the undeformed models. The average volume fractions of crack obtained for the models $\boldsymbol{A}, \boldsymbol{G}$ and $\boldsymbol{D}$ are $15.24 \%, 17.32 \%$ and $18.08 \%$, respectively, which implies that the irregular aggregates induce a greater damage volume and the angular polyhedral Voronoi particles have the most effect on it. It should be mentioned that no difference was noted between the volume of the failure cloud of the models for the uniaxial loading case. Also, as stated earlier, the CDP model is not able to provide precise data on the crack dimensions. However, the results can be used for comparison in this study by relying on the same approach to calculate the total volume of cracks for all models. Besides, further analysis of the dissipated energy versus shape parameters shown below will confirm this interpretation.

\subsection{Stress-strain response}

Heretofore, the crack mechanisms of concrete under uniaxial and biaxial compression have been analysed based on visualisation. Hereafter, the damage of concrete is first evaluated using stressstrain behaviour during loading time. Then, the values of the critical stresses and the dissipated energy against the shape parameters are presented and discussed in detail. The total amount of irreversible loss of energy is calculated by the sum of the energy dissipated by the plastic deformation (ALLPD) and the damage (ALLDMD) in mortar and ITZ as the output variables available in ABAQUS.

Fig. 19a and $\mathrm{b}$ shows the representative stress-strain curves and the corresponding standard deviations for all the sets under uniaxial and biaxial loading conditions, respectively. To facilitate the 
comparison, the stress values were normalised to the compressive strength of mortar. By comparison between the models $\boldsymbol{A}, \boldsymbol{D}$ and $\boldsymbol{G}$, the stress-strain curves are similar for both loading conditions in the elastic region. For the uniaxial loading case, the most deviation $(\sim 0.08 \mathrm{MPa})$ is found around the peak points (between Points I and III) that are shown in the magnified subplots, while the softening branches stay the same. At the peak point (Point II), the standard deviation of each curve is maximum and varies from about 0.03 to $0.045 \mathrm{MPa}$. So, it may be noted that the peak stress (i.e. compressive strength) does not change significantly and generally and the compressive behaviour of concrete remains unchanged with different type of aggregates in view of stress-strain response. For the biaxial loading case, the deviation can also be observed after the peak points during the softening branch, but it seems that models are less predictable in comparison with the uniaxial loading case due to the relatively higher standard deviations. The values are greater than $0.1 \mathrm{MPa}$ around the peak points $(0.0006<\varepsilon<0.0017)$ and they are decreased to $0.025 \mathrm{MPa}$. Besides, the materials seem to be more brittle under this loading condition since the stress level drastically reduces after the peak point. Hence, a characteristic point such as Point III would not be recognised easily because of the fast growth of the crack in the whole system. The comparison between the models $\boldsymbol{B}$ to $\boldsymbol{D}$ and $\boldsymbol{E}$ to $\boldsymbol{G}$ reveals that by increasing the irregularity level, the peak stress decreases about $10 \%$ and $20 \%$ under uniaxial and biaxial loading, respectively. As expected, the material also becomes more homogenous as the standard deviation reduces, especially for the biaxial cases. Moreover, the brittleness increases since the strain values at the peak stress is reduced and the range between Points I and III gets smaller.

More specifically, the effect of the shape parameters is analysed by plotting the critical values of stress and the dissipated energy against the mean values of elongation ratio, sphericity and roundness for all the sets as depicted in Fig. 20. Based on the comparison presented in Table 2, the data points related to the groups of $(\boldsymbol{B}, \boldsymbol{C}, \boldsymbol{D})$ and $(\boldsymbol{E}, \boldsymbol{F}, \boldsymbol{G})$ are connected. By following the dotted lines in Fig. 20 , it can be observed that the stress and the energy values are reduced by decreasing the shape parameters for each group, which suggests that the increase of irregularity level can result in degradation of material's strength and fracture toughness. With a general view of the models $\boldsymbol{B}$ to $\boldsymbol{G}$, no specific relationship can be found with respect to the shape parameters, except for the uniaxial loading case, the relation of the elongation ratio and the dissipated energy, which shows a similar data trend to the stress at $\varepsilon=0.002$. The dissipated energy proportionally changes with the elongation ratio in the range of 0.3 to 0.7 . This can be explained by the fact that a structure with less elongation is comprised of some small local surface area, which causes enhancement of stress concentration and consequently damage initiation as discussed earlier. Regarding this, the previous study [73] also reported that the elongation ratio as a main factor has a significant influence on the macroscopic mechanical property of concrete. Although, it cannot be expanded for the biaxial loading case based on the current numerical results that the dissipated energy seems to be sensitive to other parameters 
with different comparison of data. Earlier, the volume of crack cluster was related to the dissipated energy under biaxial loading through the comparison of the group $(\boldsymbol{A}, \boldsymbol{D}, \boldsymbol{G})$. Here, it can be observed that the dissipated energy increases about $20 \%$, when the sphericity and roundness values decrease from 1 to about 0.7 . In view of simulation, the model $\boldsymbol{A}$ with the spherical aggregates underestimates the dissipated energy, while the model $\boldsymbol{D}$ with the polyhedral Voronoi aggregates overestimates it. Therefore, the model $\boldsymbol{G}$ with splined-Voronoi aggregates can moderately predict the material behaviour. It implies that the angular aggregates like crushed rocks would induce more damage compared to the rounded one like natural gravel. Furthermore, in relation to sphericity and roundness, the data tendency shows the influence of splining. This can be realised by looking at the increment of the critical stress and the energy in the groups of $(\boldsymbol{B}, \boldsymbol{E}),(\boldsymbol{C}, \boldsymbol{F}),(\boldsymbol{G}, \boldsymbol{D})$ as these shape parameters increase. As demonstrated earlier in Fig. 5, the total average of sphericity and roundness level in the mesostructure are higher for the splined-Voronoi aggregates.

In summary, the mechanical response of concrete under uniaxial and biaxial compression in terms of dissipated energy was found to be highly dependent on the shape parameters according to the description presented above, whilst the compressive strength shows less dependency on them. However, it is known that in the real world, the class of angular aggregates can enhance the interlocking effect and consequently compressive strength and fracture toughness in comparison with the rounded aggregates [74]. It is due to the interaction of several inherent physical/structural characteristics of the angular aggregates including (1) rough surface, which can cause a stronger bond between aggregates and matrix, (2) possible higher strength of aggregates, (3) higher convexity, (4) lower elongation ratio, and (5) higher angularity [75-79]. In the present models, the local roughness of the aggregate surface is disregarded. Also, the material properties assigned for aggregate and ITZ (i.e. bond strength) are similar for all models. Therefore, the compressive strength does not significantly change by the change of shape among models $\boldsymbol{A}, \boldsymbol{D}$ and $\boldsymbol{G}$, while the post-failure behaviour is more sensitive to the shape parameters.

\section{Conclusions}

In this study, a novel mesostructure model of concrete consisting of coarse aggregates, mortar and interfacial transition zone (ITZ) between them accounting for its mesostructural characteristics such as the content, random location, size distribution and shape of aggregate was developed. Based on the generated 3D mesostructure, the effect of aggregate shape on fracture process in concrete under uniaxial and biaxial compression was investigated in a quantitative manner using the finite element continuum damage plasticity model. The main conclusions can be drawn as follows:

- The compressive strength of concrete is strongly influenced by the random location and size distribution of aggregates while their shape characteristics have an insignificant effect, indicating that the $3 \mathrm{D}$ mesostructure models of concrete with the simplified aggregate geometries such as 
sphere and polyhedron would be able to properly predict the compressive strength.

- The crack initiation in concrete is highly related to two main structural factors that would significantly influence the local stress concentration, i.e., the small distance among the aggregates, and the irregular nature of aggregates such as the small local surface area and sharp edges and corners. Therefore, a mesostructure model with spherical aggregates cannot simulate the effect of the second factor due to their perfectly round shape. Also, a model with polyhedral aggregates may not yield reliable results due to the aggregate shape with high unnatural angularity and flatness. For a comprehensive analysis, it is important to note that the stress variations do not clearly reflect the damage induced by the second factor, especially under uniaxial compression. Hence, the dissipated energy and crack evolution should also be investigated to capture this effect.

- Generally, the increment of irregularity level of aggregates in terms of shape and location can facilitate the spread of damage and crack growth in different directions, which consequently changes the compressive fragmentation mode by breaking samples to smaller pieces. Under biaxial compression, the volume fraction of the crack clusters and dissipated energy that can represent the damage level are greater for concrete with irregularly shaped aggregates.

- Regarding the relationship between the damage and shape parameters, the dissipated energy is sensitive to the elongation ratio under uniaxial loading. Moreover, this energy increases with the decrease of the values of sphericity and roundness in the biaxial loading case, where the cracking process is more complex. This indicates the importance of these parameters and further demonstrates the advantage of the shape adjustability of the models.

To sum up, the aggregate irregularity has a significant influence on concrete fracture and should be taken into account for concrete mix design, as the concrete durability is highly related to the crack initiation and growth. For future research, it is vital to develop a suitable meso-scale fracture model that can provide accurate information on the crack width while considering the inner frictional force and voids. This is a subject of ongoing work and will be presented in a future publication.

\section{Acknowledgements}

The authors gratefully acknowledge the financial support from the Engineering and Physical Sciences Research Council (EPSRC) under Grant Nos. EP/R041504/1 and1836739 as well as the Royal Society under Award No. IEC\NSFC $\backslash 191417$ and the British Council under Award No. 352639234. The financial support provided by University College London (UCL) to the second author is also gratefully acknowledged.

\section{References}

[1] M. Zhang, Multiscale lattice Boltzmann-finite element modelling of transport properties in cement-base materials, PhD Thesis, Delft University of Technology, Delft 2013.

[2] A. Gangnant, J. Saliba, C. La Borderie, S. Morel, Modeling of the quasibrittle fracture of concrete 
at meso-scale: Effect of classes of aggregates on global and local behavior, Cement and Concrete Research, 89 (2016) 35-44.

[3] R.Z. Al-Rousan, M.A. Alhassan, E.A. AlShuqari, Behavior of plain concrete beams with DSSF strengthened in flexure with anchored CFRP sheets-Effects of DSSF content on the bonding length of CFRP sheets, Case Studies in Construction Materials, 9 (2018) e00195.

[4] P.E. Petersson, Fracture Energy of Concrete - Practical Performance and Experimental Results, Cement and Concrete Research, 10 (1980) 91-101.

[5] T. Holušová, S. Seitl, A. Fernández-Canteli, Numerical support of experimental compact tension test on concrete cylindric specimens, Transactions of the VŠB-Technical University of Ostrava, Civil Engineering Series, 13 (2013) 31-40.

[6] P.-E. Petersson, Crack growth and development of fracture zones in plain concrete and similar materials, University of Lund, Sweden, (1981).

[7] F. Mujika, On the difference between flexural moduli obtained by three-point and four-point bending tests, Polymer Testing, 25 (2006) 214-220.

[8] F. Bencardino, L. Rizzuti, G. Spadea, R.N. Swamy, Implications of test methodology on postcracking and fracture behaviour of Steel Fibre Reinforced Concrete, Composites Part B: Engineering, 46 (2013) 31-38.

[9] R.X. Zhou, Z.H. Song, Y. Lu, 3D mesoscale finite element modelling of concrete, Computers \& Structures, 192 (2017) 96-113.

[10] W. Ren, Z. Yang, R. Sharma, C. Zhang, P.J. Withers, Two-dimensional X-ray CT image based meso-scale fracture modelling of concrete, Engineering Fracture Mechanics, 133 (2015) 24-39.

[11] C.M. López, I. Carol, A. Aguado, Meso-structural study of concrete fracture using interface elements. I: numerical model and tensile behavior, Materials and Structures, 41 (2008) 583-599.

[12] X. Xi, S. Yang, C.-Q. Li, A non-uniform corrosion model and meso-scale fracture modelling of concrete, Cement and Concrete Research, 108 (2018) 87-102.

[13] E. Karavelić, M. Nikolić, A. Ibrahimbegovic, A. Kurtović, Concrete meso-scale model with full set of 3D failure modes with random distribution of aggregate and cement phase. Part I: Formulation and numerical implementation, Computer Methods in Applied Mechanics and Engineering, 344 (2019) 1051-1072.

[14] P. Thilakarathna, K.K. Baduge, P. Mendis, E. Chandrathilaka, V. Vimonsatit, H. Lee, Understanding fracture mechanism and behaviour of ultra-high strength concrete using mesoscale modelling, Engineering Fracture Mechanics, 234 (2020) 107080.

[15] O. Yilmaz, J.F. Molinari, A mesoscale fracture model for concrete, Cement and Concrete Research, 97 (2017) 84-94.

[16] K. Sobolev, S. Shah, Nanotechnology of concrete: recent developments and future perspectives, 
ACI 2008.

[17] K. Sobolev, Nanotechnology and nanoengineering of construction materials, Nanotechnology in Construction, Springer 2015, pp. 3-13.

[18] X. Wang, M. Zhang, A.P. Jivkov, Computational technology for analysis of 3D meso-structure effects on damage and failure of concrete, International Journal of Solids and Structures, 80 (2016) 310-333.

[19] S. Bhowmik, S. Ray, An experimental approach for characterization of fracture process zone in concrete, Engineering Fracture Mechanics, 211 (2019) 401-419.

[20] J.P. Heath, J.H. Harding, D.C. Sinclair, J.S. Dean, Electric field enhancement in ceramic capacitors due to interface amplitude roughness, Journal of the European Ceramic Society, 39 (2019) 1170-1177.

[21] M. Ghasemi, M.R. Ghasemi, S.R. Mousavi, Investigating the effects of maximum aggregate size on self-compacting steel fiber reinforced concrete fracture parameters, Construction and Building Materials, 162 (2018) 674-682.

[22] V. Afroughsabet, T. Ozbakkaloglu, Mechanical and durability properties of high-strength concrete containing steel and polypropylene fibers, Construction and Building Materials, 94 (2015) 73-82.

[23] J.Q. Chen, H. Wang, H.C. Dan, Y.J. Xie, Random modeling of three-dimensional heterogeneous microstructure of asphalt concrete for mechanical analysis, Journal of Engineering Mechanics, 144 (2018) 04018083.

[24] S. Thomas, Y. Lu, E.J. Garboczi, Improved model for three-dimensional virtual concrete: Anm model, Journal of Computing in Civil Engineering, 30 (2016) 04015027.

[25] S. Naderi, M. Zhang, An integrated framework for modelling virtual 3D irregulate particulate mesostructure, Powder Technology, 355 (2019) 808-819.

[26] Y. Liu, F.Y. Gong, Z.P. You, H.N. Wang, Aggregate morphological characterization with 3D optical scanner versus X-ray computed tomography, Journal of Materials in Civil Engineering, 30 (2018) 04017248.

[27] P. Carrara, R. Kruse, D.P. Bentz, M. Lunardelli, T. Leusmann, P.A. Varady, L. De Lorenzis, Improved mesoscale segmentation of concrete from 3D X-ray images using contrast enhancers, Cement \& Concrete Composites, 93 (2018) 30-42.

[28] G. Sokhansefat, M.T. Ley, M.D. Cook, R. Alturki, M. Moradian, Investigation of concrete workability through characterization of aggregate gradation in hardened concrete using X-ray computed tomography, Cement and Concrete Composites, 98 (2019) 150-161.

[29] D. Wei, R.C. Hurley, L.H. Poh, D. Dias-da-Costa, Y. Gan, The role of particle morphology on concrete fracture behaviour: A meso-scale modelling approach, Cement and Concrete Research, 134 
(2020) 106096.

[30] Y. Peng, L. Ying, M.M. Kamel, Y. Wang, Mesoscale fracture analysis of recycled aggregate concrete based on digital image processing technique, Structural Concrete, (2020) 1-15.

[31] G. Mazzucco, B. Pomaro, G. Xotta, C.E. Maiorana, V.A. Salomoni, Tomography reconstruction of concrete materials for mesoscale modelling, Engineering Computations, 37 (2020) 2275-2291.

[32] T. Liu, S. Qin, D. Zou, W. Song, J. Teng, Mesoscopic modeling method of concrete based on statistical analysis of CT images, Construction and Building Materials, 192 (2018) 429-441.

[33] Z. Gyurko, R. Nemes, Fracture modelling of normal concrete using different types of aggregates, Engineering Failure Analysis, 101 (2019) 464-472.

[34] N. Benkemoun, P. Poullain, H. Al Khazraji, M. Choinska, A. Khelidj, Meso-scale investigation of failure in the tensile splitting test: Size effect and fracture energy analysis, Engineering fracture mechanics, 168 (2016) 242-259.

[35] G. Chen, Y.F. Hao, H. Hao, 3D meso-scale modelling of concrete material in spall tests, Materials and Structures, 48 (2015) 1887-1899.

[36] Y. Zhang, Q. Chen, Z. Wang, J. Zhang, Z. Wang, Z. Li, 3D mesoscale fracture analysis of concrete under complex loading, Engineering Fracture Mechanics, 220 (2019) 106646.

[37] T. Saksala, Numerical modelling of concrete fracture processes under dynamic loading: Mesomechanical approach based on embedded discontinuity finite elements, Engineering Fracture Mechanics, 201 (2018) 282-297.

[38] Y.-Q. Huang, S.-W. Hu, Y.-Y. Sun, The Effect of Inner Friction on Concrete Fracture Behavior under Biaxial Compression: A 3D Mesostructure Study, Materials, 12 (2019) 3880.

[39] Q. Xiong, X. Wang, A.P. Jivkov, A 3D multi-phase meso-scale model for modelling coupling of damage and transport properties in concrete, Cement and Concrete Composites, 109 (2020) 103545. [40] J.M. Wang, A.P. Jivkov, D.L. Engelberg, Q.M. Li, Meso-scale modelling of mechanical behaviour and damage evolution in normal strength concrete, ECF22 - Loading and Environmental Effects on Structural Integrity, 13 (2018) 560-565.

[41] M. Khormani, V.R.K. Jaari, I. Aghayan, S.H. Ghaderi, A. Ahmadyfard, Compressive strength determination of concrete specimens using X-ray computed tomography and finite element method, Construction and Building Materials, 256 (2020) 119427.

[42] Y. Huang, Z. Yang, W. Ren, G. Liu, C. Zhang, 3D meso-scale fracture modelling and validation of concrete based on in-situ X-ray Computed Tomography images using damage plasticity model, International Journal of Solids and Structures, 67 (2015) 340-352.

[43] J. Wang, A.P. Jivkov, D.L. Engelberg, Q. Li, Meso-scale modelling of mechanical behaviour and damage evolution in normal strength concrete, Procedia Structural Integrity, 13 (2018) 560-565.

[44] H. Chen, B. Xu, Y. Mo, T. Zhou, Behavior of meso-scale heterogeneous concrete under uniaxial 
tensile and compressive loadings, Construction and Building Materials, 178 (2018) 418-431.

[45] D.T. Hashim, F. Hejazi, V.Y. Lei, Simplified Constitutive and Damage Plasticity Models for UHPFRC with Different Types of Fiber, International Journal of Concrete Structures and Materials, $14(2020) 1-21$.

[46] M. Wimalasiri, D. Robert, C.-Q. Li, Permeability degradation of stressed concrete considering concrete plasticity, Journal of Materials in Civil Engineering, 32 (2020) 04020265.

[47] J.J. Huang, Q. Peng, X.B. Hu, Y.X. Du, A combined-alpha-shape-implicit-surface approach to generate 3D random concrete mesostructures via digital image processing, spectral representation, and point cloud, Construction and Building Materials, 143 (2017) 330-365.

[48] Y.H. Zhang, Q.Q. Chen, Z.Y. Wang, J. Zhang, Z.H. Wang, Z.Q. Li, 3D mesoscale fracture analysis of concrete under complex loading, Engineering Fracture Mechanics, 220 (2019) 106646.

[49] M. Zhang, A.P. Jivkov, Micromechanical modelling of deformation and fracture of hydrating cement paste using X-ray computed tomography characterisation, Composites Part B: Engineering, 88 (2016) 64-72.

[50] T. Lv, X. Chen, G. Chen, The 3D meso-scale model and numerical tests of split Hopkinson pressure bar of concrete specimen, Construction and Building Materials, 160 (2018) 744-764.

[51] R. Zhou, Z. Song, Y. Lu, 3D mesoscale finite element modelling of concrete, Computers \& Structures, 192 (2017) 96-113.

[52] F.-Y. Wang, M.-L. Zhou, D.-M. Zhang, H.-W. Huang, D. Chapman, Random evolution of multiple cracks and associated mechanical behaviors of segmental tunnel linings using a multiscale modeling method, Tunnelling and Underground Space Technology, 90 (2019) 220-230.

[53] H. Ma, L. Song, W. Xu, A novel numerical scheme for random parameterized convex aggregate models with a high-volume fraction of aggregates in concrete-like granular materials, Computers \& Structures, 209 (2018) 57-64.

[54] W. Xu, Z. Lv, H. Chen, Effects of particle size distribution, shape and volume fraction of aggregates on the wall effect of concrete via random sequential packing of polydispersed ellipsoidal particles, Physica A: Statistical Mechanics and its Applications, 392 (2013) 416-426.

[55] H. Ma, W. Xu, Y. Li, Random aggregate model for mesoscopic structures and mechanical analysis of fully-graded concrete, Computers \& Structures, 177 (2016) 103-113.

[56] J. Hu, P. Stroeven, Shape characterisation of particles in concrete technology, Proceedings of the 6th International Symposium on Cement \& Concrete and CANMET/ACI International Symposium on Concrete Technology for Sustainable Development, Vols 1 and 2, (2006) 929-937.

[57] J.W. Bullard, E.J. Garboczi, Defining shape measures for 3D star-shaped particles: Sphericity, roundness, and dimensions, Powder Technology, 249 (2013) 241-252.

[58] T.M. Al Rousan, Characterization of aggregate shape properties using a computer automated 
system, $\mathrm{PhD}$ thesis, Texas A\&M University, 2005.

[59] T.J. Hirsch, Modulus of elasticity of concrete affected by elastic moduli of cement paste matrix and aggregate, Journal Proceedings, 1962, pp. 427-452.

[60] Z.G. Tu, Y. Lu, Mesoscale modelling of concrete for static and dynamic response analysis Part 1: model development and implementation, Structural Engineering and Mechanics, 37 (2011) 197213.

[61] S.M. Kim, R.K. Abu Al-Rub, Meso-scale computational modeling of the plastic-damage response of cementitious composites, Cement and Concrete Research, 41 (2011) 339-358.

[62] J. Xiao, W. Li, D.J. Corr, S.P. Shah, Effects of interfacial transition zones on the stress-strain behavior of modeled recycled aggregate concrete, Cement and Concrete Research, 52 (2013) 82-99. [63] R.R. Pedersen, A. Simone, L.J. Sluys, Mesoscopic modeling and simulation of the dynamic tensile behavior of concrete, Cement and Concrete Research, 50 (2013) 74-87.

[64] T. Long, H.E. Zhang, Y. Chen, Z. Li, J.G. Xu, X.S. Shi, Q.Y. Wang, Effect of sulphate attack on the flexural fatigue behaviour of fly ash-based geopolymer concrete, Journal of Strain Analysis for Engineering Design, 53 (2018) 711-718.

[65] Y.J. Huang, Z.J. Yang, W.Y. Ren, G.H. Liu, C.Z. Zhang, 3D meso-scale fracture modelling and validation of concrete based on in-situ X-ray Computed Tomography images using damage plasticity model, International Journal of Solids and Structures, 67-68 (2015) 340-352.

[66] X.L. Du, L. Jin, G.W. Ma, Numerical simulation of dynamic tensile-failure of concrete at mesoscale, International Journal of Impact Engineering, 66 (2014) 5-17.

[67] A. De Sam, R.S. Deepa, Studies on ambient cured geopolymer concrete, Emerging Trends in Engineering, Science and Technology for Society, Energy and Environment, (2018) 3-6.

[68] H.B. Chen, B. Xu, Y. Mo, T.M. Zhou, Behavior of meso-scale heterogeneous concrete under uniaxial tensile and compressive loadings, Construction and Building Materials, 178 (2018) 418-431. [69] Y. Sümer, M. Aktaş, Defining parameters for concrete damage plasticity model, Challenge Journal of Structural Mechanics, 1 (2015) 149-155.

[70] S. Gao, Nonlinear finite element failure analysis of bolted steel-concrete composite frame under column-loss, Journal of Constructional Steel Research, 155 (2019) 62-76.

[71] L.N. Lowes, Finite element modeling of reinforced concrete beam-column bridge connections, $\mathrm{PhD}$ thesis, University of California, Berkeley 1999.

[72] Z.H. Song, Y. Lu, Mesoscopic analysis of concrete under excessively high strain rate compression and implications on interpretation of test data, International Journal of Impact Engineering, 46 (2012) 41-55.

[73] Y.L. Zhou, H. Jin, B.L. Wang, Modeling and mechanical influence of meso-scale concrete considering actual aggregate shapes, Construction and Building Materials, 228 (2019) 116785. 
[74] M. Kaplan, Flexural and compressive strength of concrete as affected by the properties of coarse aggregates, Journal Proceedings, 55 (1959) 1193-1208.

[75] M. Garg, S. Singh, S. Singh, Comparative study of M35 and M40 grades of concrete by ACI, DOE, USBR and BIS methods of mix design using rounded aggregate, (2015).

[76] G. Giaccio, R. Zerbino, Failure mechanism of concrete: combined effects of coarse aggregates and strength level, Advanced Cement Based Materials, 7 (1998) 41-48.

[77] T. Özturan, C. Çeçen, Effect of coarse aggregate type on mechanical properties of concretes with different strengths, Cement and Concrete Research, 27 (1997) 165-170.

[78] J. Tay, K. Show, S. Hong, Reuse of industrial sludge as construction aggregates, Water Science and Technology, 44 (2001) 269-272.

[79] G. Guinea, K. El-Sayed, C. Rocco, M. Elices, J. Planas, The effect of the bond between the matrix and the aggregates on the cracking mechanism and fracture parameters of concrete, Cement and Concrete Research, 32 (2002) 1961-1970. 


\section{Graphical abstract}

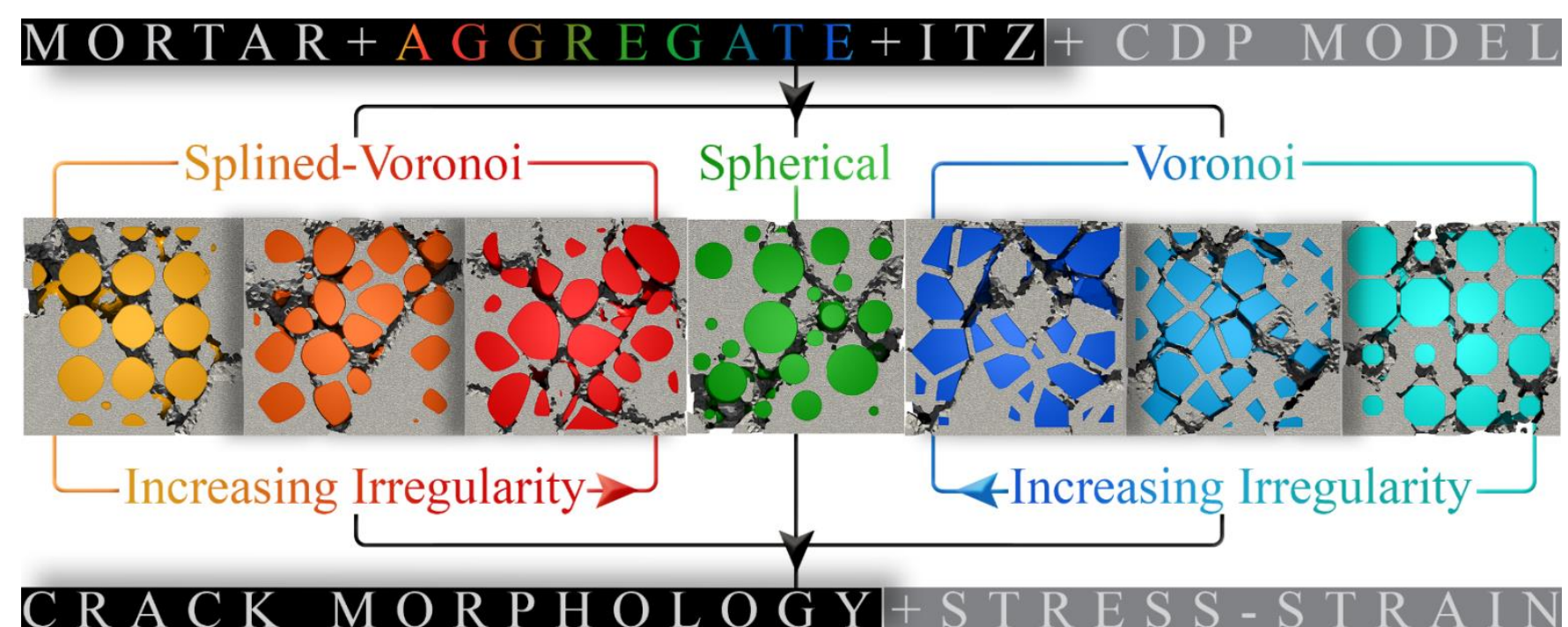




\section{Increasing Irregularity}
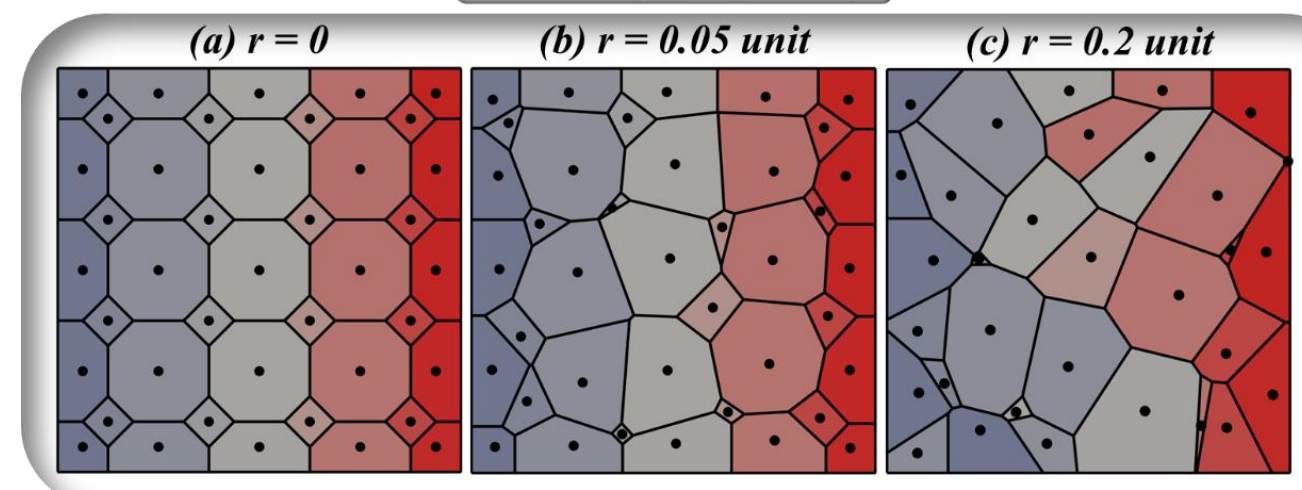

Fig. 1. Change in shape of the Voronoi cells from regular (a) to irregular (b) and (c) with increasing maximum length of vector $\vec{r}$, which indicates the displacement of the seed points from their original positions in the model (a). The side length of the squares is 1 unit.

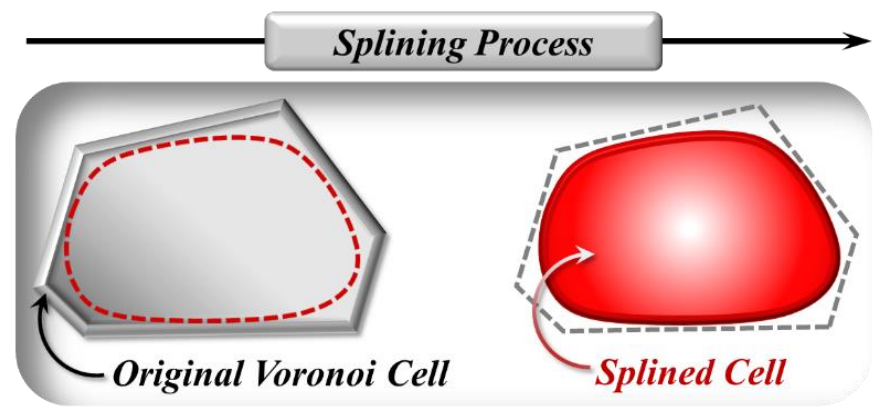

Fig. 2. Schematic illustration of the splining process where the splined cell is created through the faces of the original Voronoi cell. 


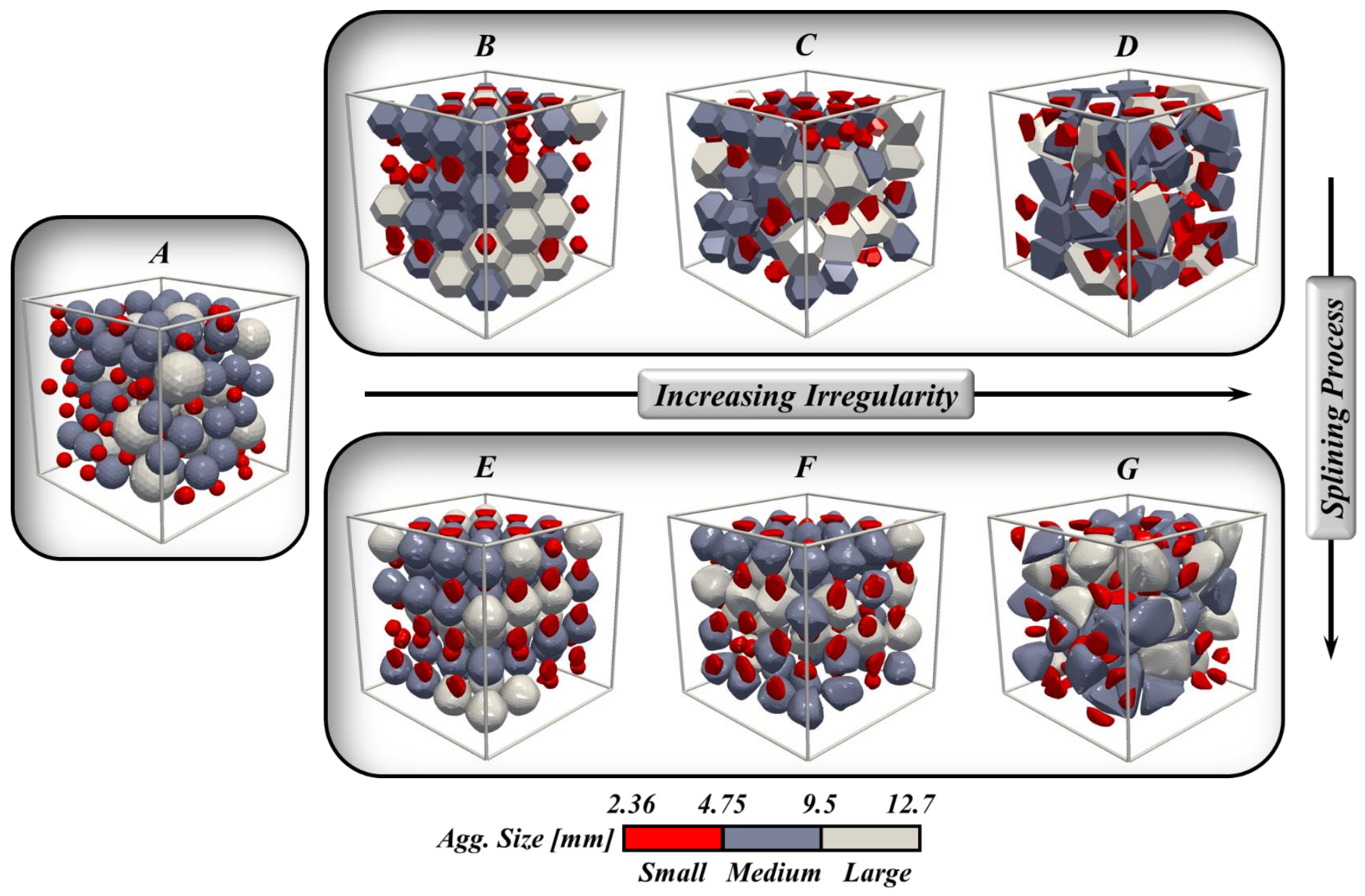

Fig. 3. Designed 3D mesoscale concrete specimens with increasing irregularity and splining process $(\boldsymbol{B}-\boldsymbol{G})$, in comparison with the reference with spherical aggregate particles $(\boldsymbol{A})$.
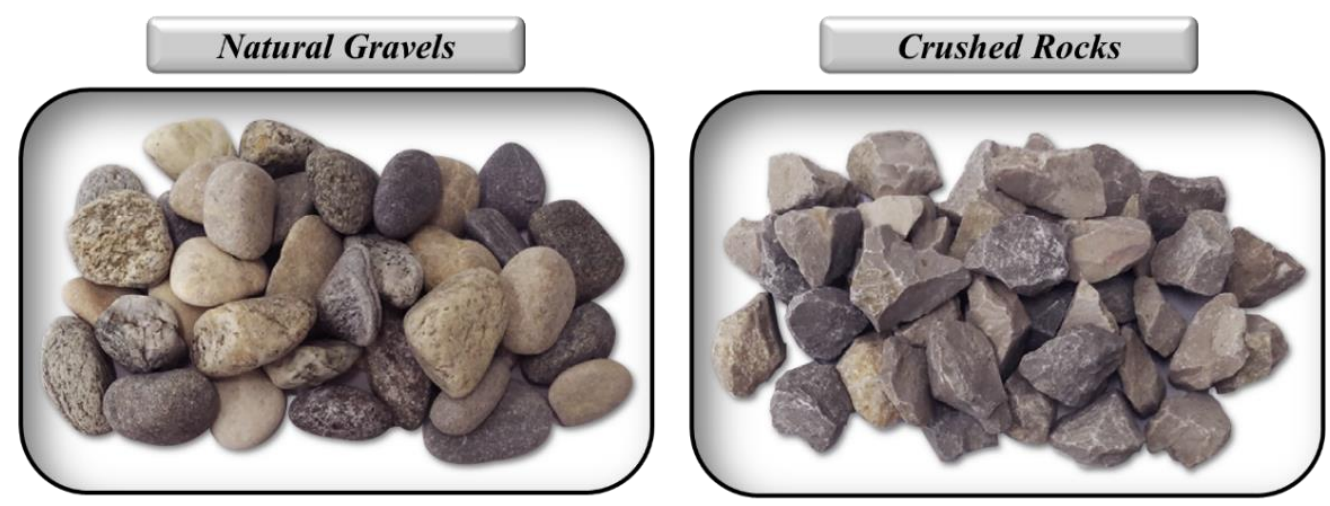

Fig. 4. Natural gravels and crushed rocks widely used as coarse aggregates in concrete which are displayed for visual comparison between real and virtual aggregates exemplified in Table 1 and Fig. 3. 

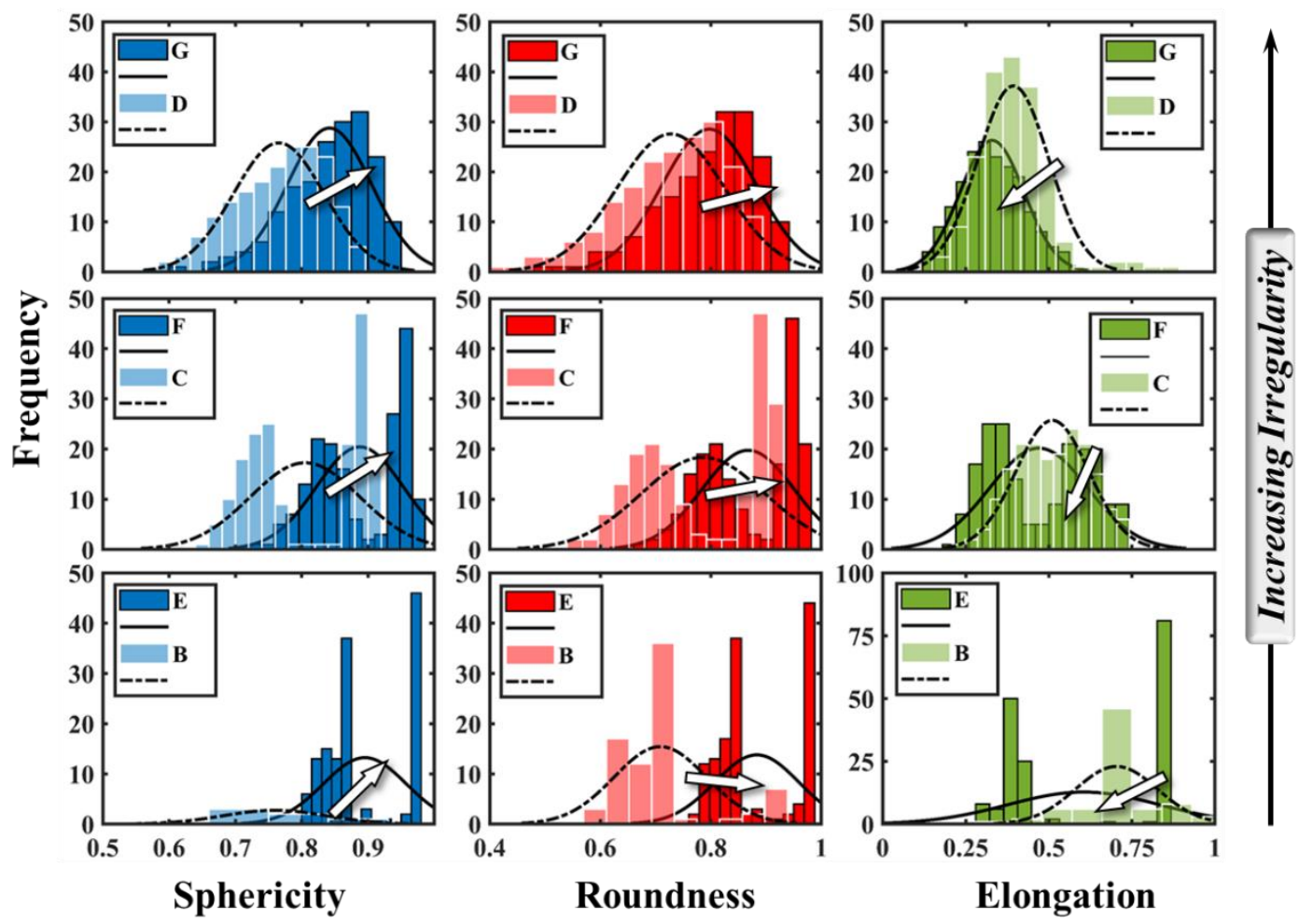

Fig. 5. Representative histograms of the shape parameters (sphericity, roundness and elongation) for models $\boldsymbol{B}$ -

$\boldsymbol{G}$. The normal probability density functions (PDFs) are fitted, which highlight the shape transformation by increasing the irregularity level and the splining process. The arrows as a guide to the eyes point to the splining effect on the PDFs. Due to overlapping regions, the results of the polyhedral Voronoi cells are shown in transparent form in each plot.

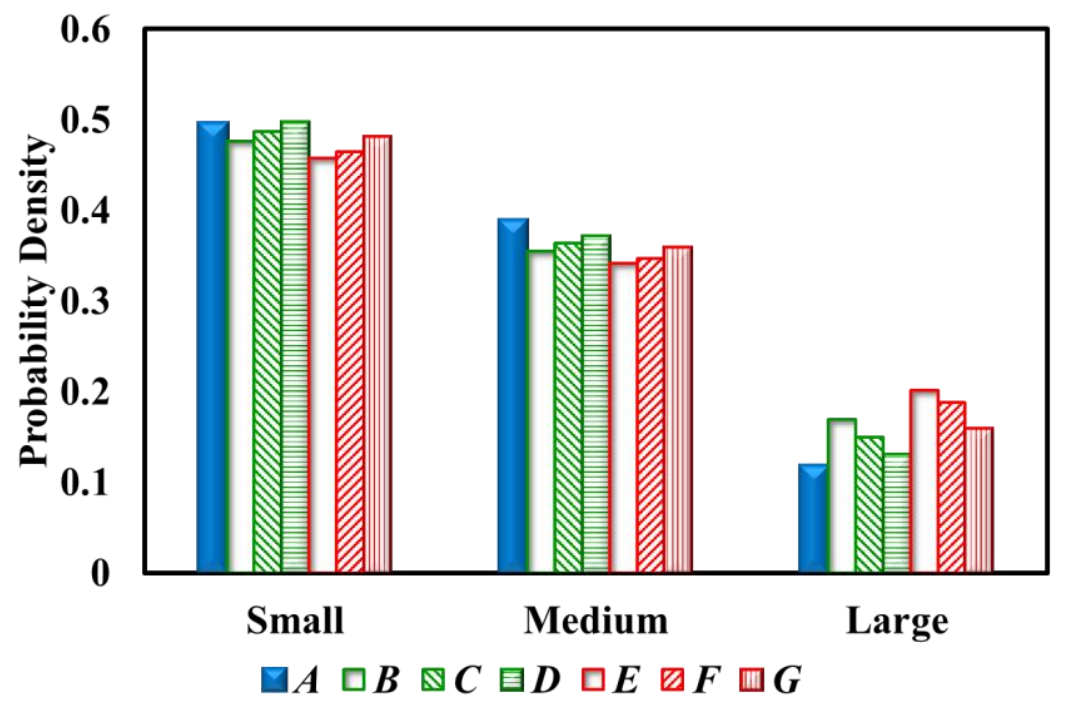

Fig. 6. Mean values of the probability density for the small, medium and large aggregates, obtained by averaging the aggregate probability density of all samples in each set per size range. The probability density represents the ratio of aggregate numbers in each size range to the total number of particles. The standard deviation for all data is less than 0.01 . 

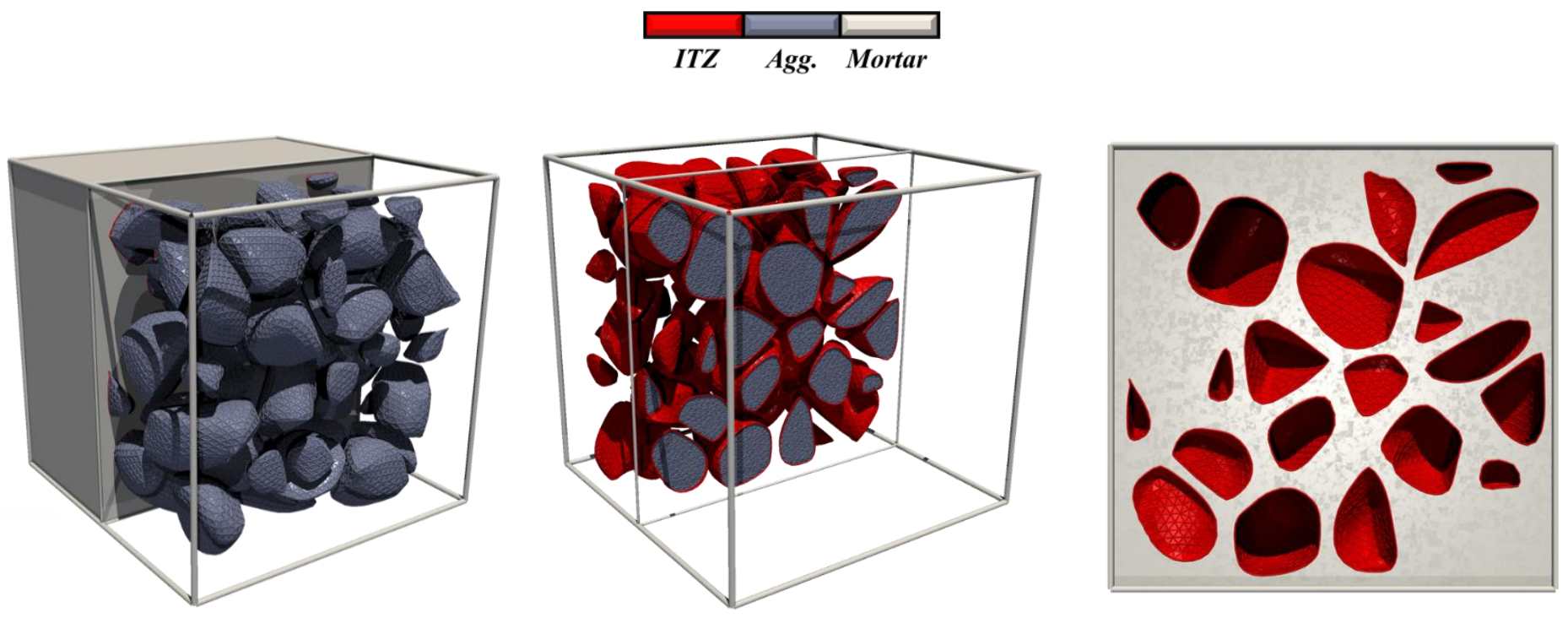

Fig. 7. Cross-sectional views of 3D mesostructure of concrete consisting of mortar matrix, aggregates and ITZs around aggregates.
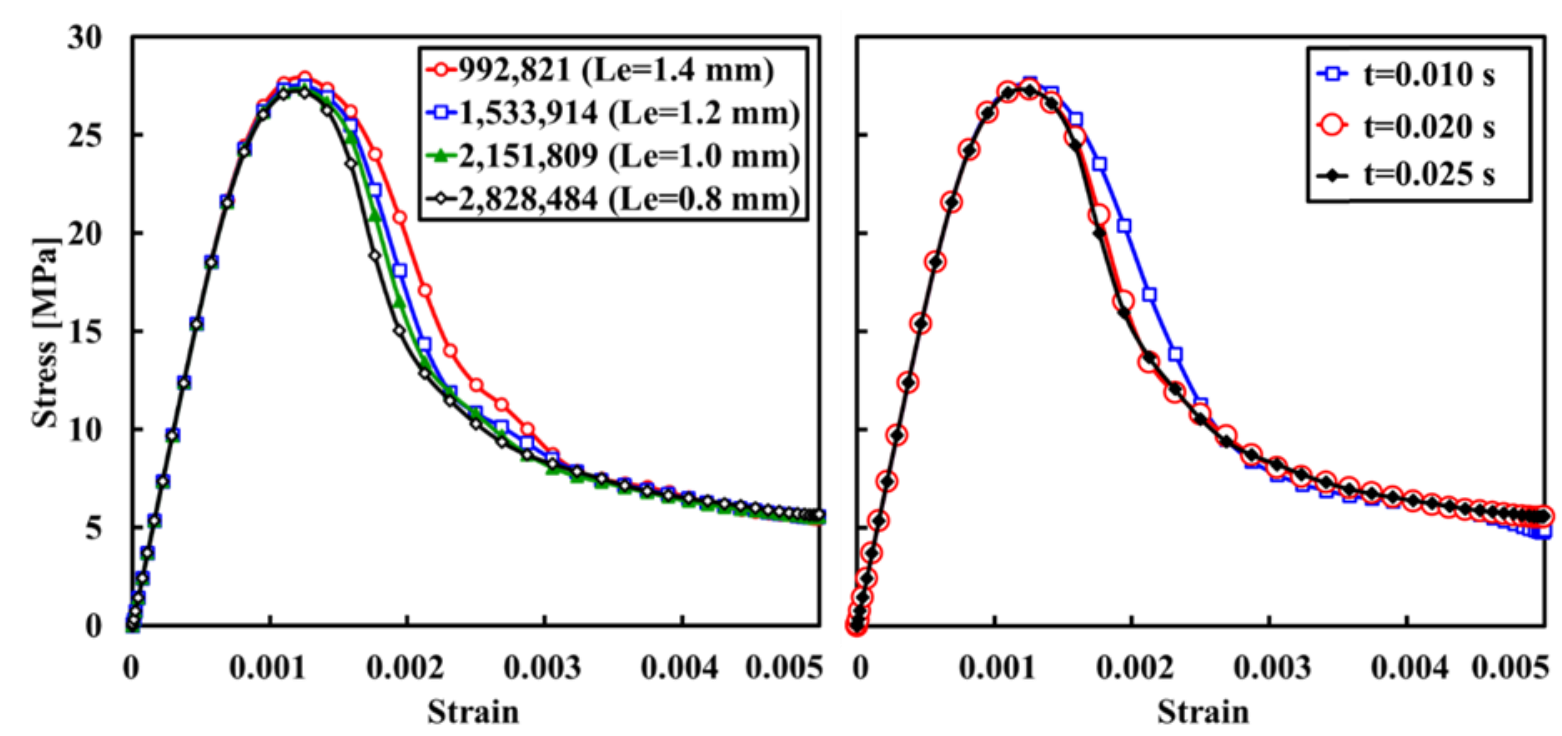

Fig. 8. Analysis of the effects of simulation parameters including element size $\left(L_{e}\right)$, element number and time step (t) on the stress-strain response based on the model $\boldsymbol{A}$ under uniaxial compression. $L_{e}$ denotes the maximum element characteristic length in RVE. 


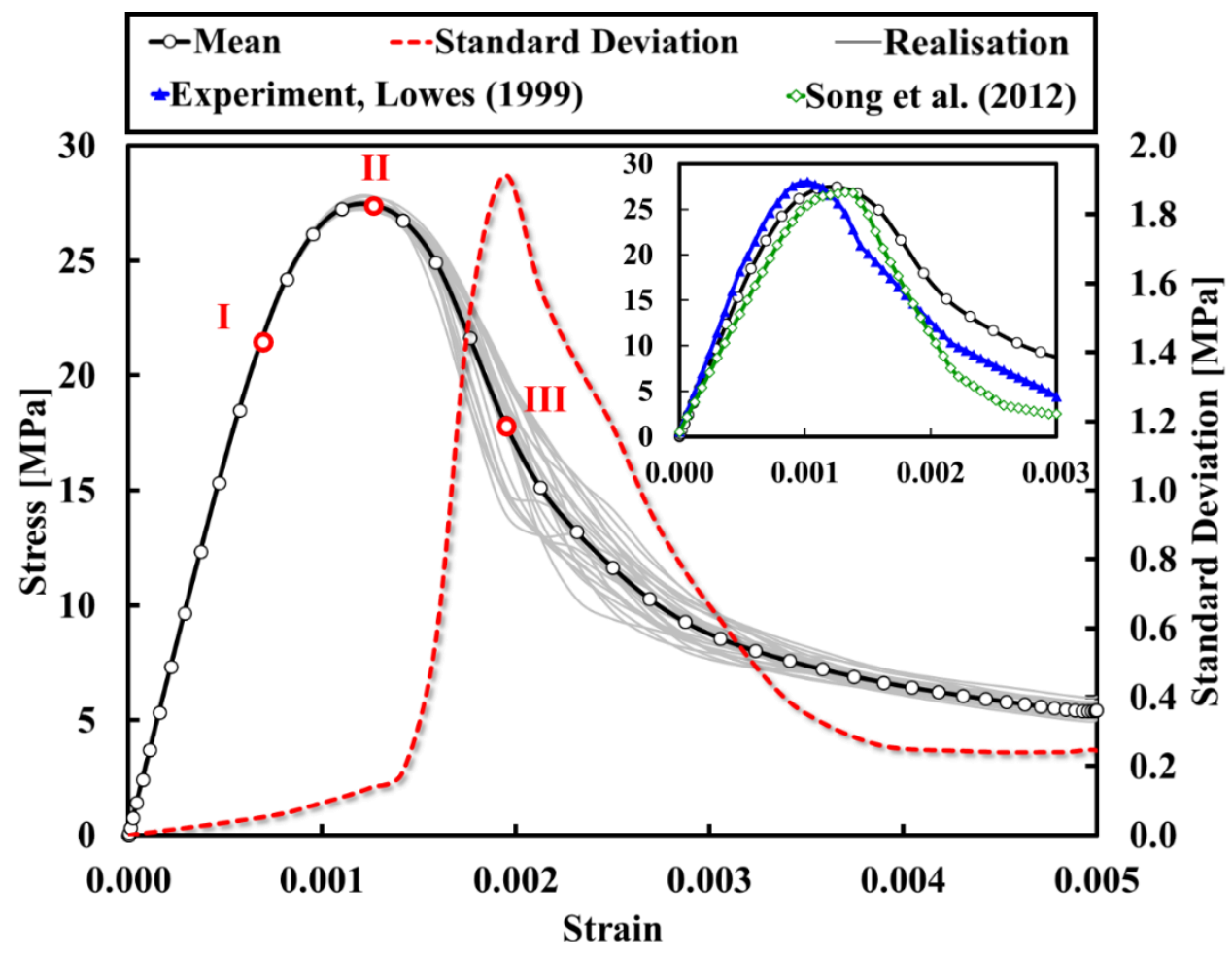

Fig. 9. Stress-strain curves obtained based on the simulations of 30 samples of the model $\boldsymbol{A}$, under uniaxial compression. The corresponding mean and standard deviation are calculated for the stress values during loading. The subplot shows the results acquired from the experiments [40] and other numerical work [41] in comparison with the mean stress curve. Points I, II and III specify the elastic region, the material strength and the critical stress at maxim standard deviation on the mean curve. 


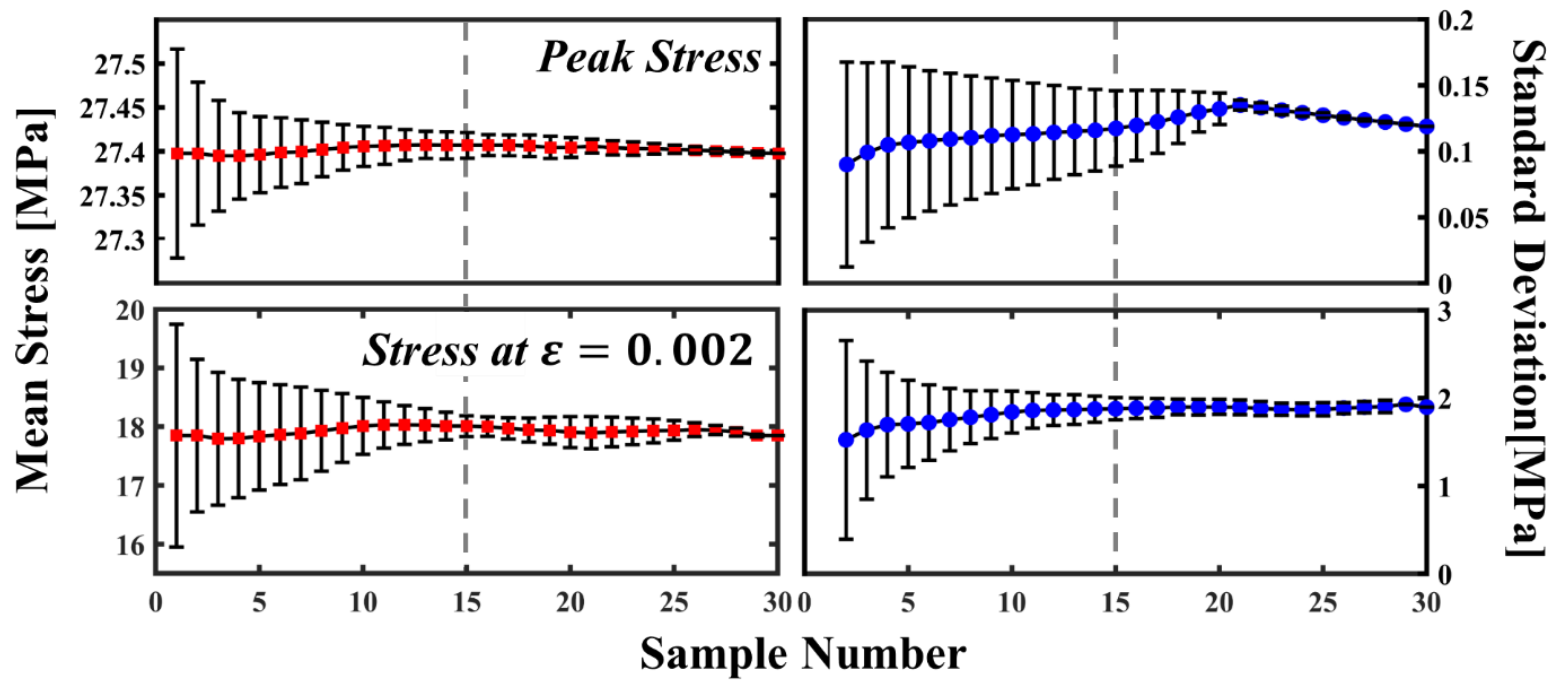

Fig. 10. Effect of sample number on the peak stress and the stress at $\varepsilon=0.002$ as the critical parameters in the point of view of statistics for the model $\boldsymbol{A}$. The means of these parameters are firstly computed for all possible combination of $n$ samples where $n \leq 30$ and the averages of the mean values are then calculated. The minimum sample number is indicated by the dashed lines where the parameters tend to become constant.
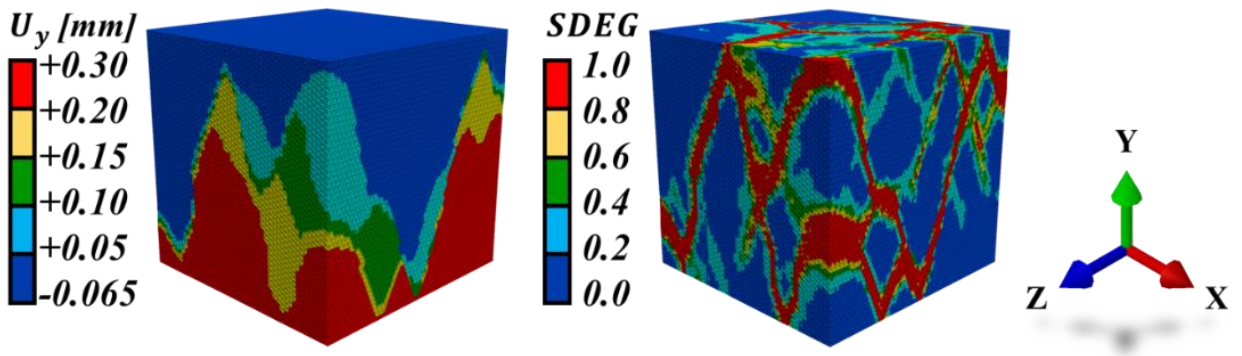

(a)
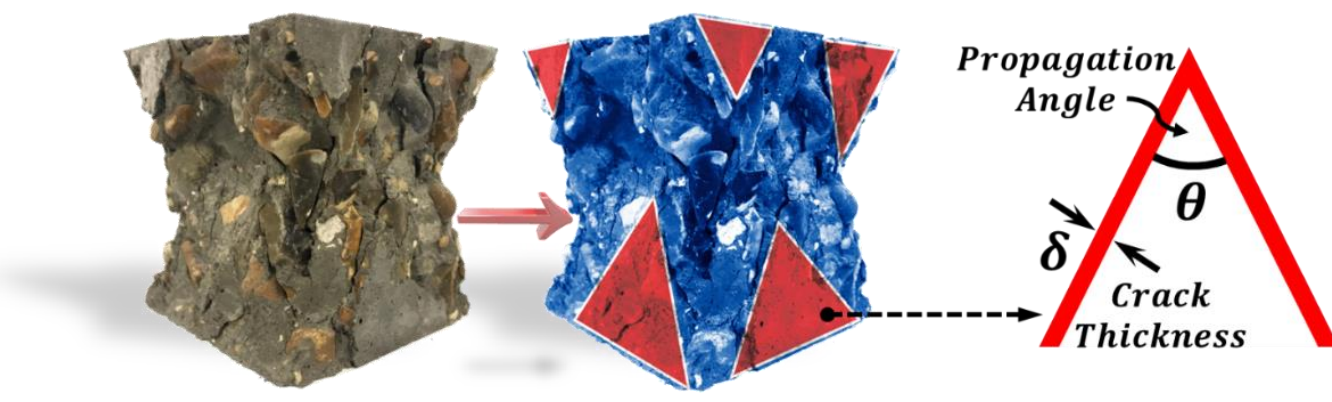

(b)

Fig. 11. Crack patterns mostly seen among the numerical models, using the displacement $\left(U_{y}\right)$ and damage (SDEG) filed distributions for the model $\boldsymbol{G}$ under unixail loading in Y-direction (a), and typical crack pattern of concrete under uniaxial compression observed in experiments (b). In both simulation and experiment, the final crack morphology can be analogized to a triangular-like structure, which is characterised by crack thickness $\delta$ and propagation angle $\theta$. 
(a)
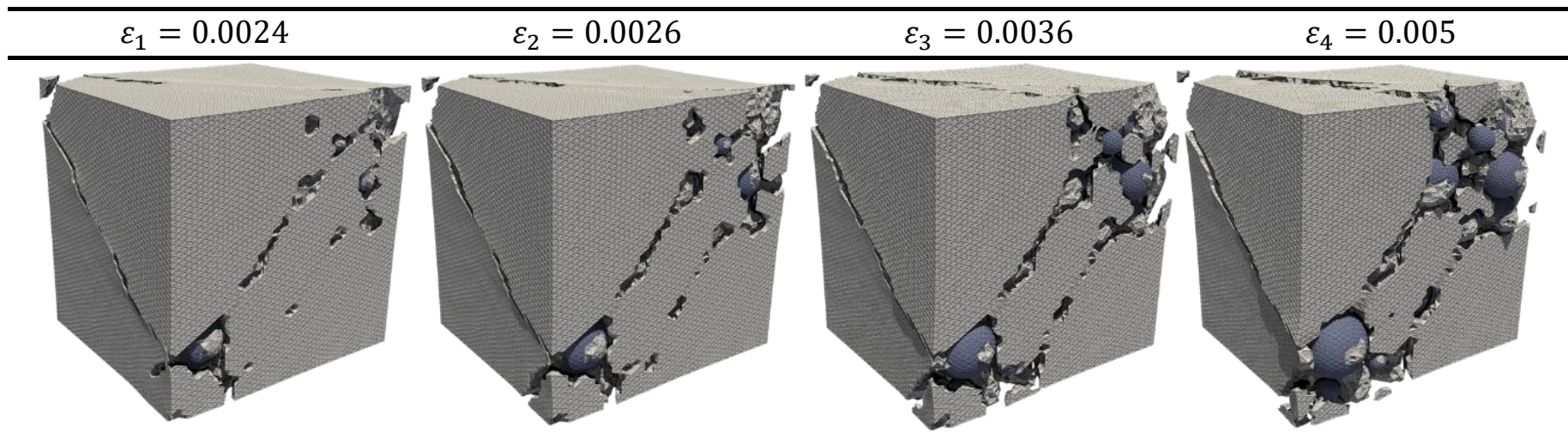

(b)
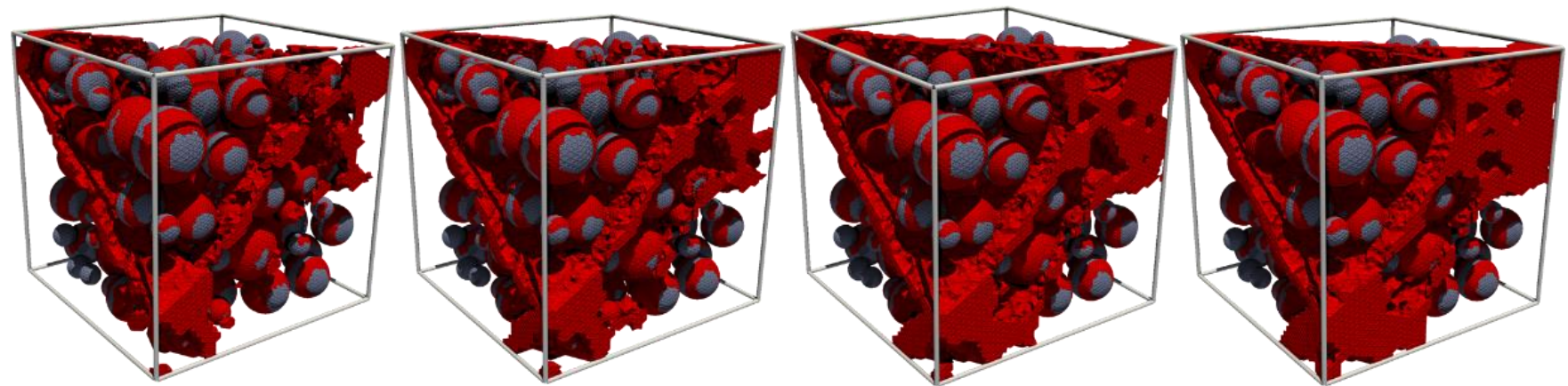

(c)
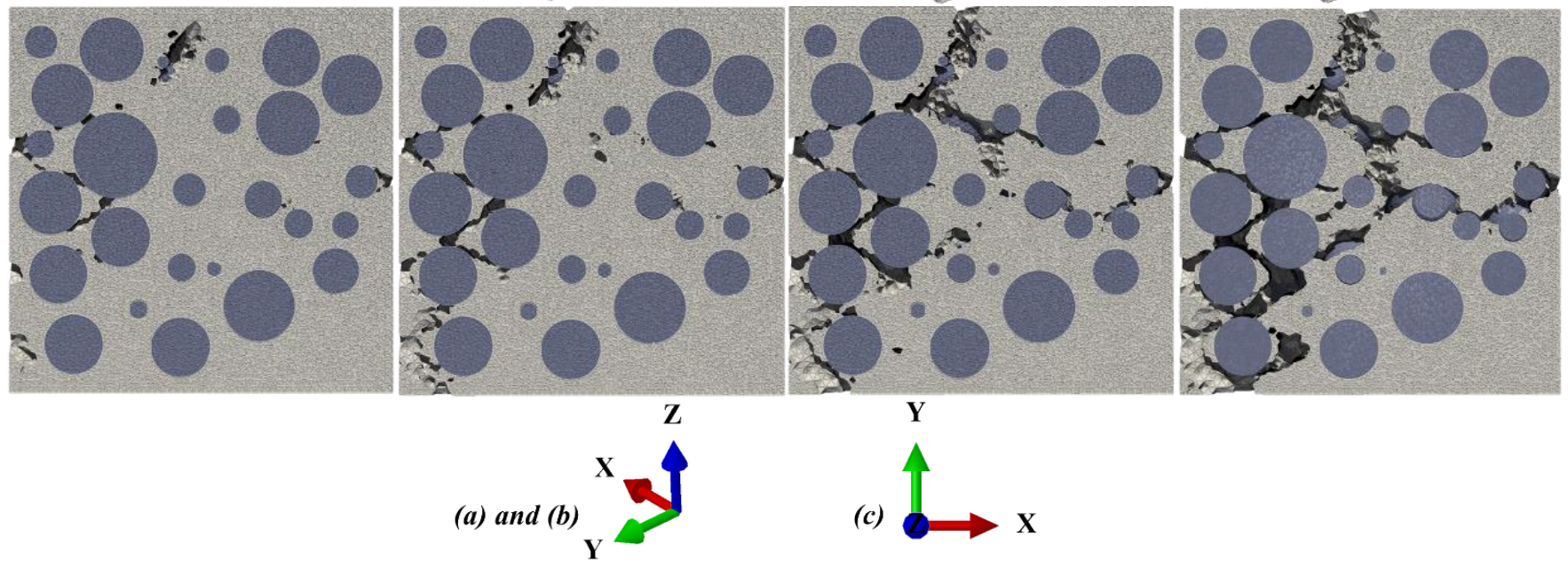

Fig. 12. Crack evolution of concrete under uniaxial compression in X-direction after about Point III $(0.002<$ $\varepsilon \leq 0.005$ ) at 4 stages for the model $\boldsymbol{A}$ in 3D (a and b) and cross-sectional view (c). The crack is visualized by deleting the corresponding elements (a and c) and the elements which SDEG values are greater than 0.9 (b). For better display, the displacements in X, Y and Z-directions are scaled by 30, 10 and 10, respectively. 
(a)
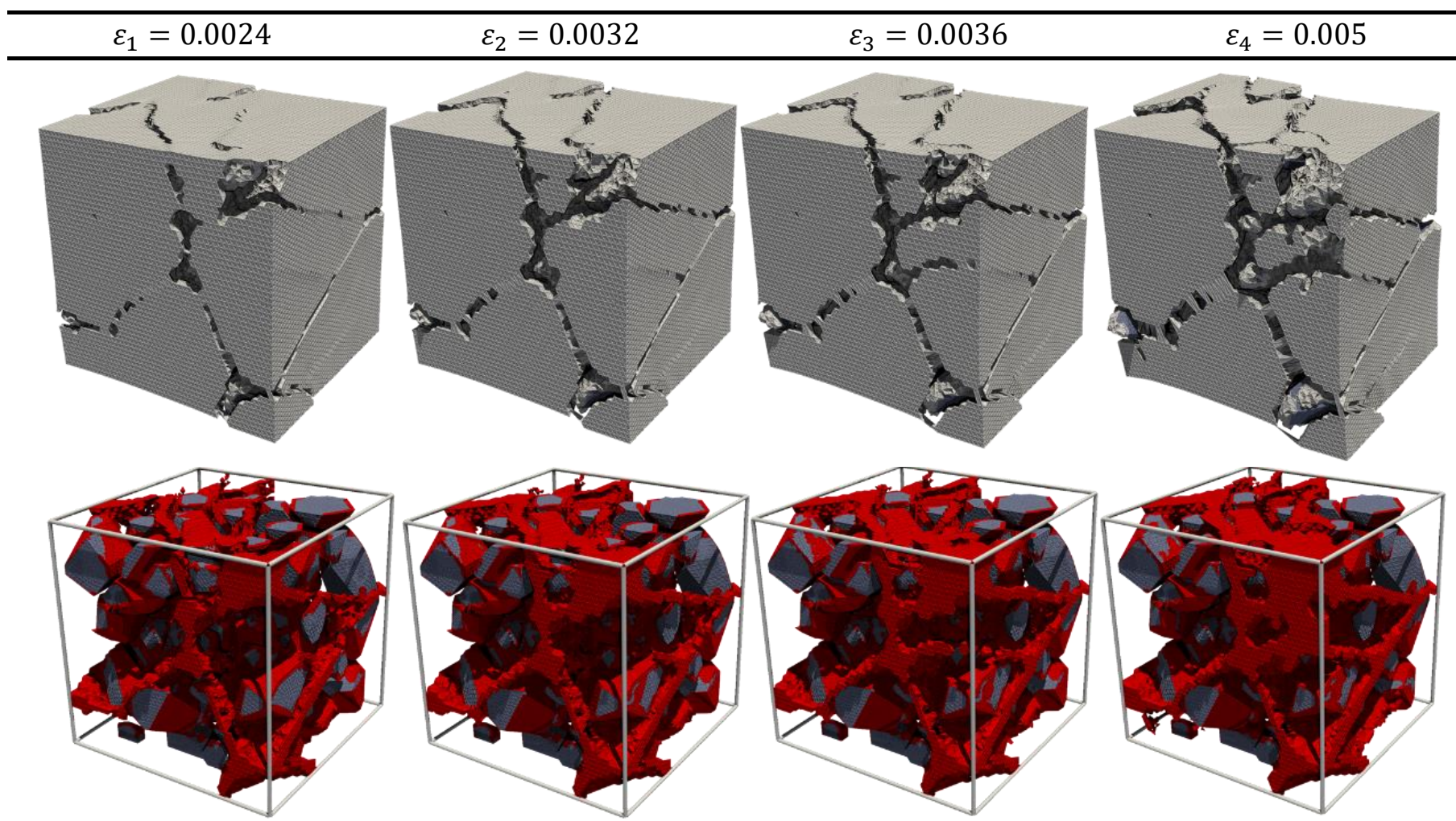

(b)
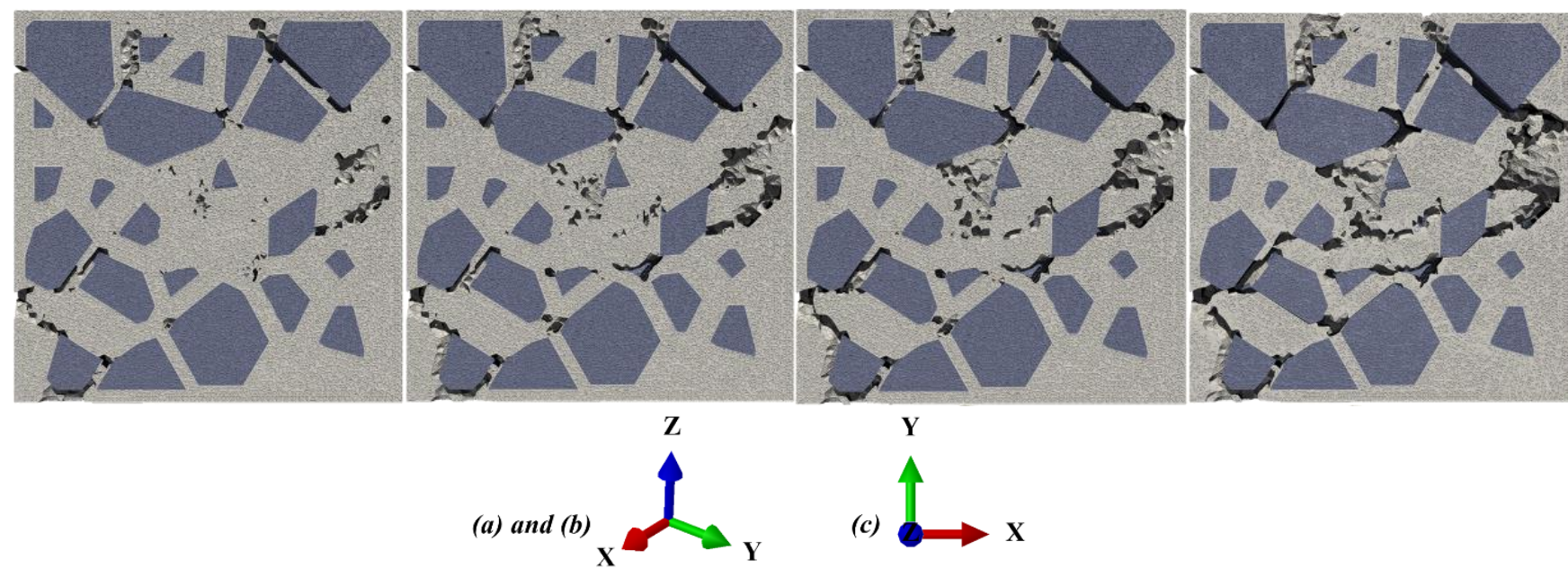

Fig. 13. Crack evolution of concrete under uniaxial compression in X-direction after about Point III $(0.002<$ $\varepsilon \leq 0.005$ ) at 4 stages for the model $\boldsymbol{D}$ in $3 \mathrm{D}$ ( $\mathrm{a}$ and b) and cross-sectional view (c). The crack is visualized by deleting the corresponding elements ( $\mathrm{a}$ and $\mathrm{c}$ ) and the elements which SDEG values are greater than 0.9 (b). For better display, the displacements in X, Y and Z-directions are scaled by 30, 10 and 10, respectively. 


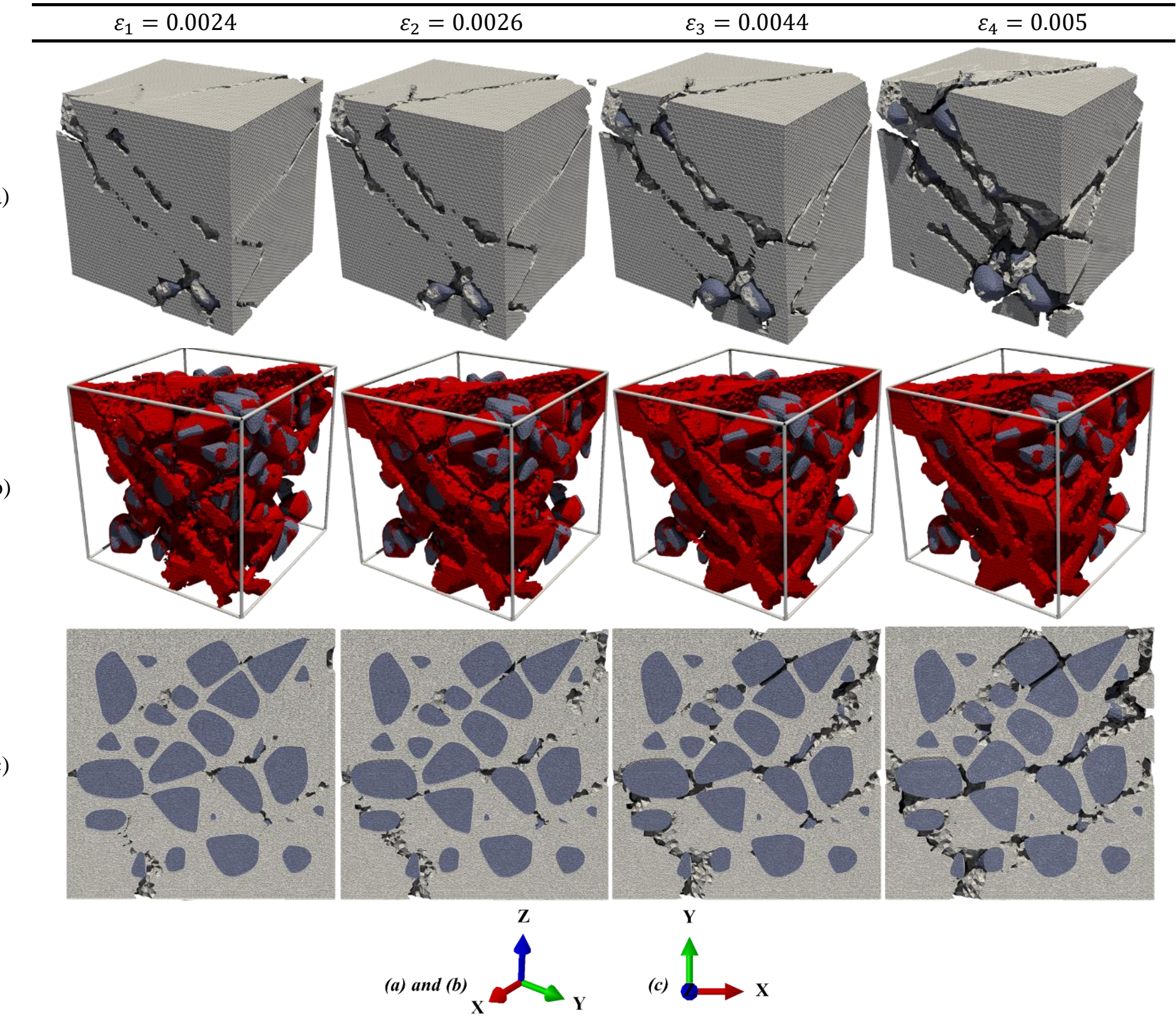

Fig. 14. Crack evolution of concrete under uniaxial compression in X-direction after about Point III $(0.002<$ $\varepsilon \leq 0.005$ ) at 4 stages for the model $\boldsymbol{G}$ in 3D (a and b) and cross-sectional view (c). The crack is visualized by deleting the corresponding elements (a and c) and the elements which SDEG values are greater than 0.9 (b). For better display, the displacements in X, Y and Z-directions are scaled by 30, 10 and 10, respectively. 


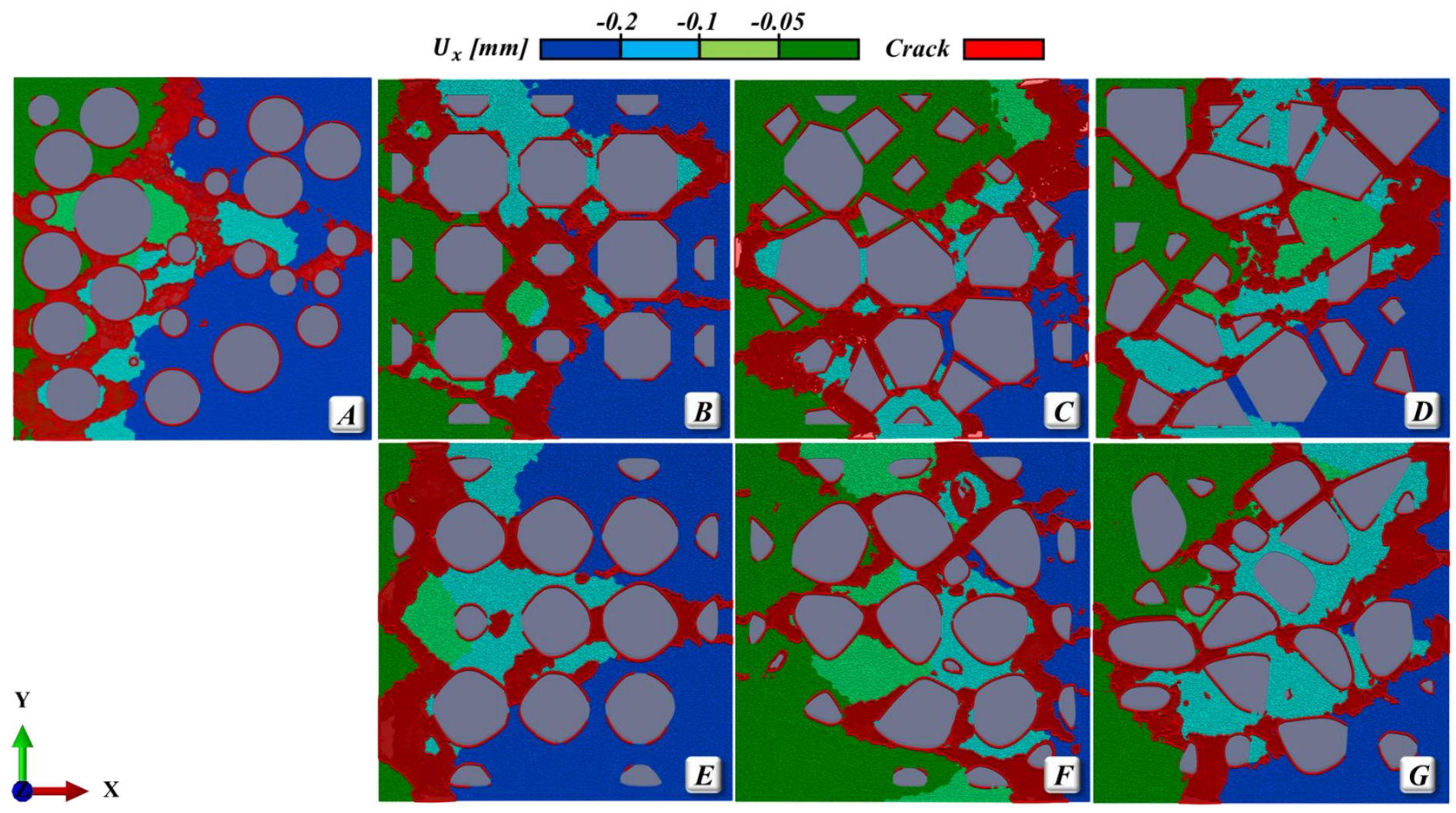

Fig. 15. Cross-sections of the displacement distribution field by the color spectrum bar and the crack in red under uniaxial compression in X-direction for all sets from $\boldsymbol{A}$ to $\boldsymbol{G}$. 
(a)

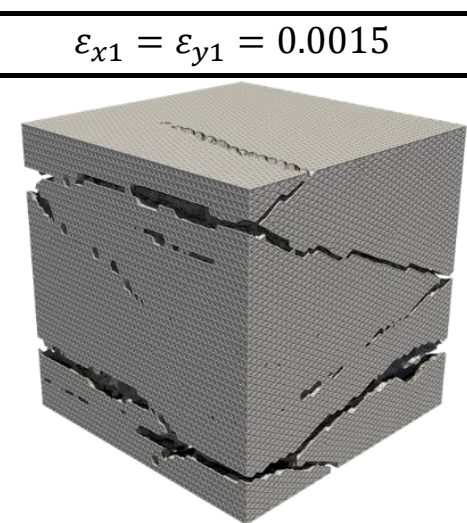

$\varepsilon_{x 2}=\varepsilon_{y 2}=0.0019$

$\varepsilon_{x 3}=\varepsilon_{y 3}=0.0022$

$\varepsilon_{x 4}=\varepsilon_{y 4}=0.004$

(b)
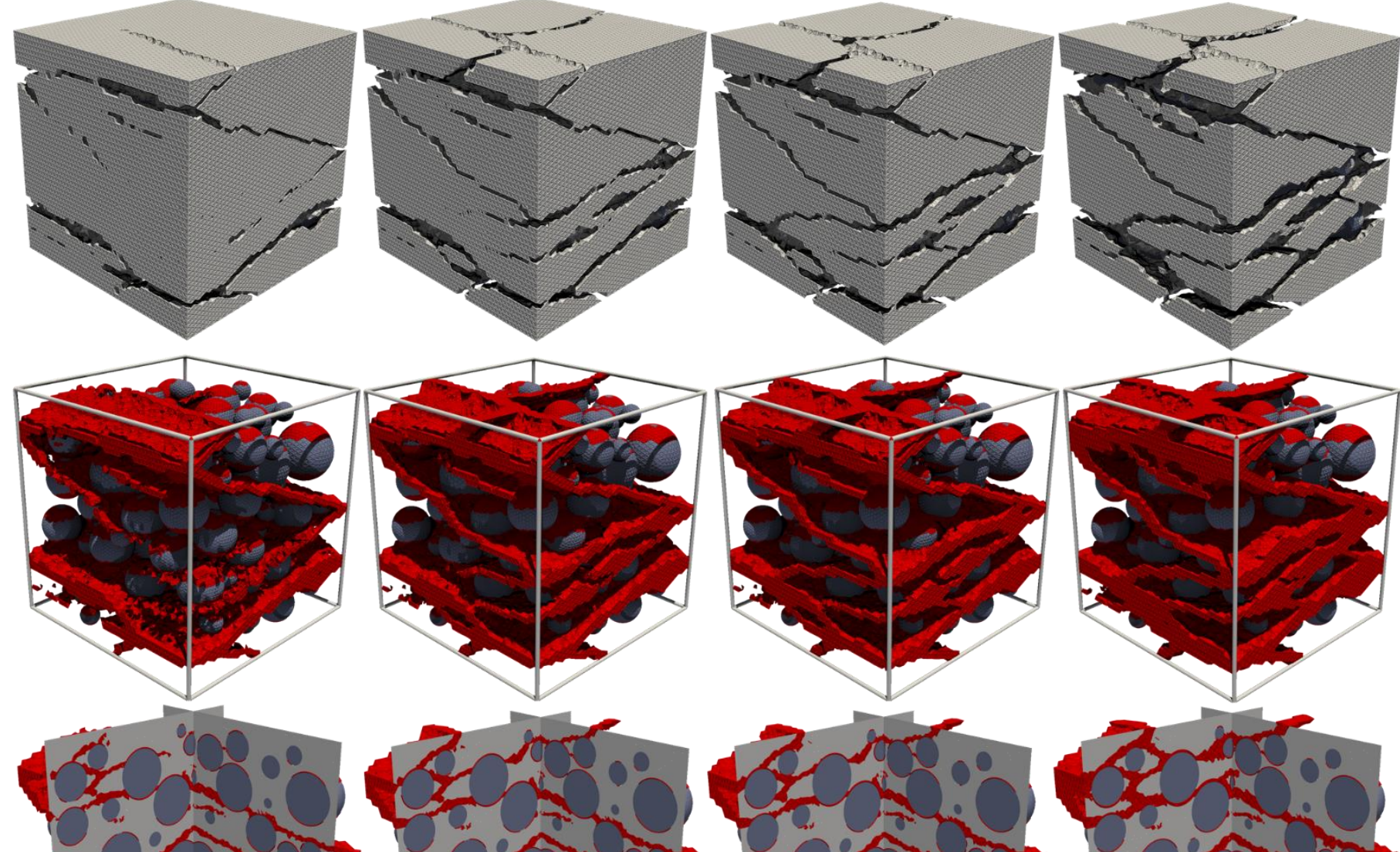

(c)
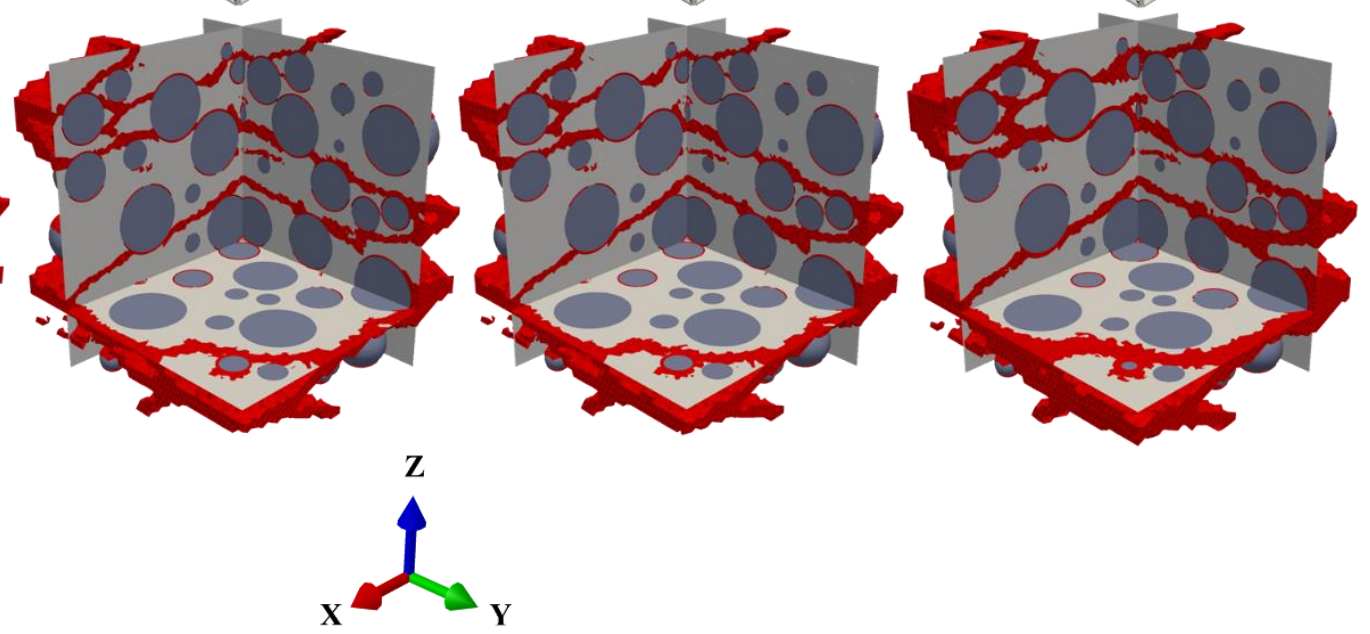

Fig. 16. Crack propagation in concrete under biaxial compression in $\mathrm{X}$ and $\mathrm{Y}$-directions where the damage is visible $(0.0015<\varepsilon \leq 0.004)$ at 4 stages for the model $\boldsymbol{A}$. The crack is visualized by deleting the corresponding elements (a) and the elements which SDEG values are greater than 0.9 (b and c). 
(a)

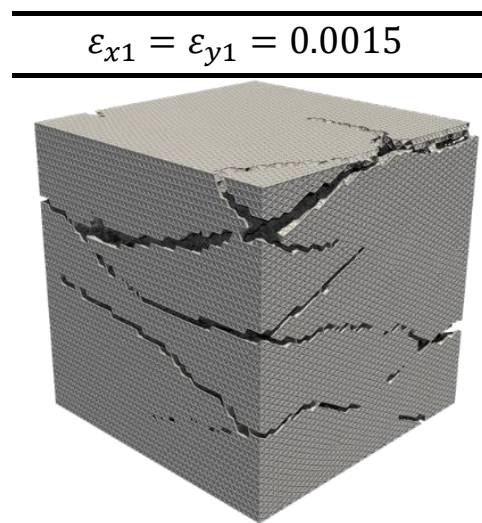

$$
\varepsilon_{x 2}=\varepsilon_{y 2}=0.0019
$$

$\varepsilon_{x 3}=\varepsilon_{y 3}=0.0022$

$\varepsilon_{x 4}=\varepsilon_{y 4}=0.004$

(b)
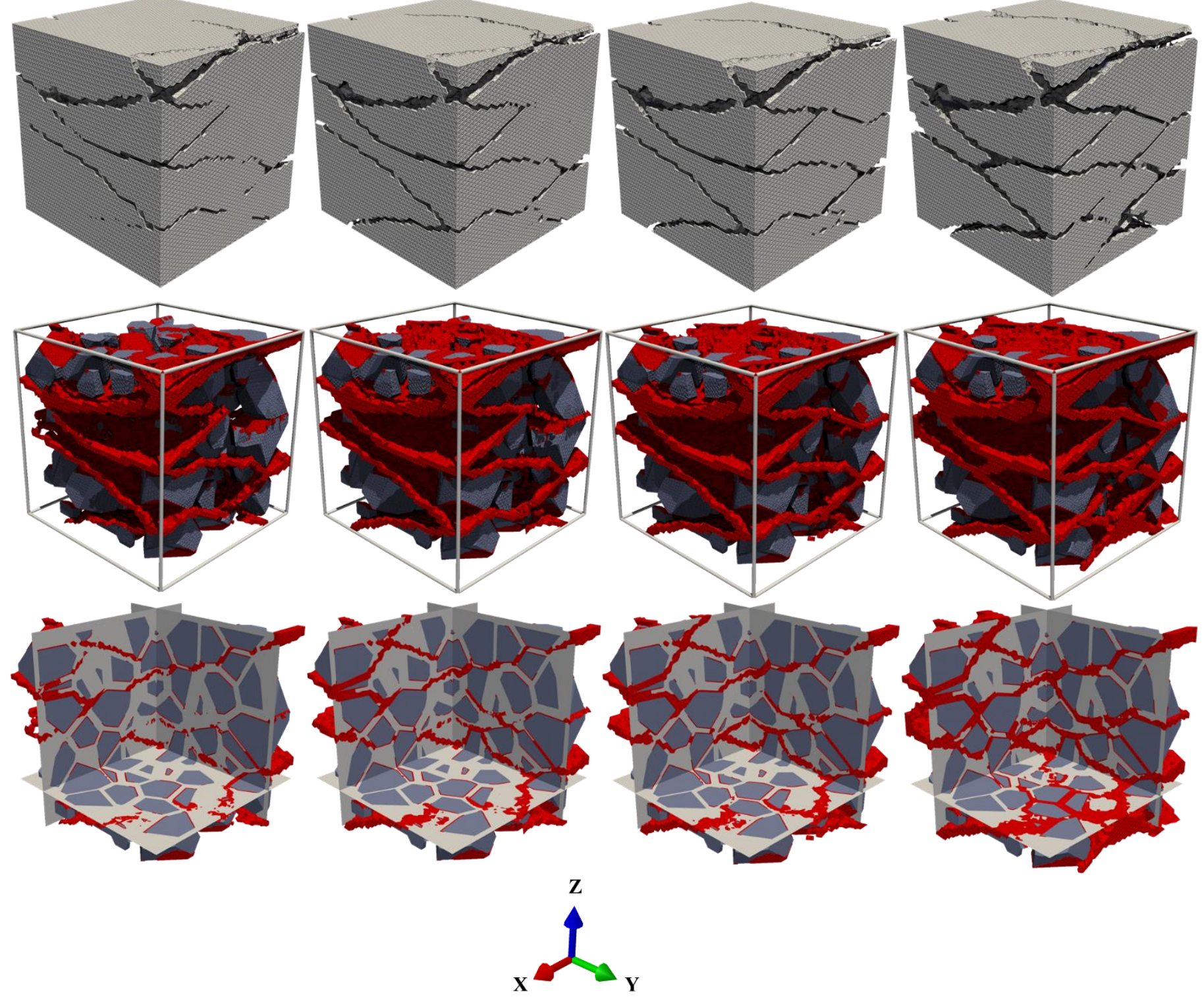

Fig. 17. Crack propagation in concrete under biaxial compression in $\mathrm{X}$ and $\mathrm{Y}$-directions where the damage is visible $(0.0015<\varepsilon \leq 0.004)$ at 4 stages for the model $\boldsymbol{D}$. The crack is visualized by deleting the corresponding elements (a) and the elements which SDEG values are greater than 0.9 (b and c). 


\begin{tabular}{llll}
\hline$\varepsilon_{x 1}=\varepsilon_{y 1}=0.0015$ & $\varepsilon_{x 2}=\varepsilon_{y 2}=0.0019$ & $\varepsilon_{x 3}=\varepsilon_{y 3}=0.0022$ & $\varepsilon_{x 4}=\varepsilon_{y 4}=0.004$ \\
\hline
\end{tabular}

(a)
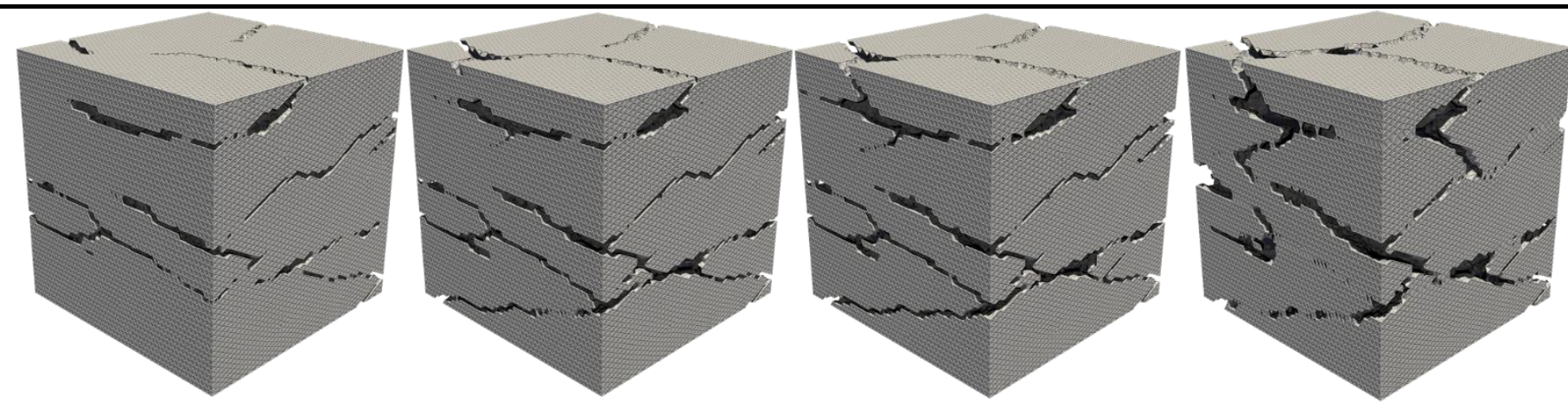

(b)
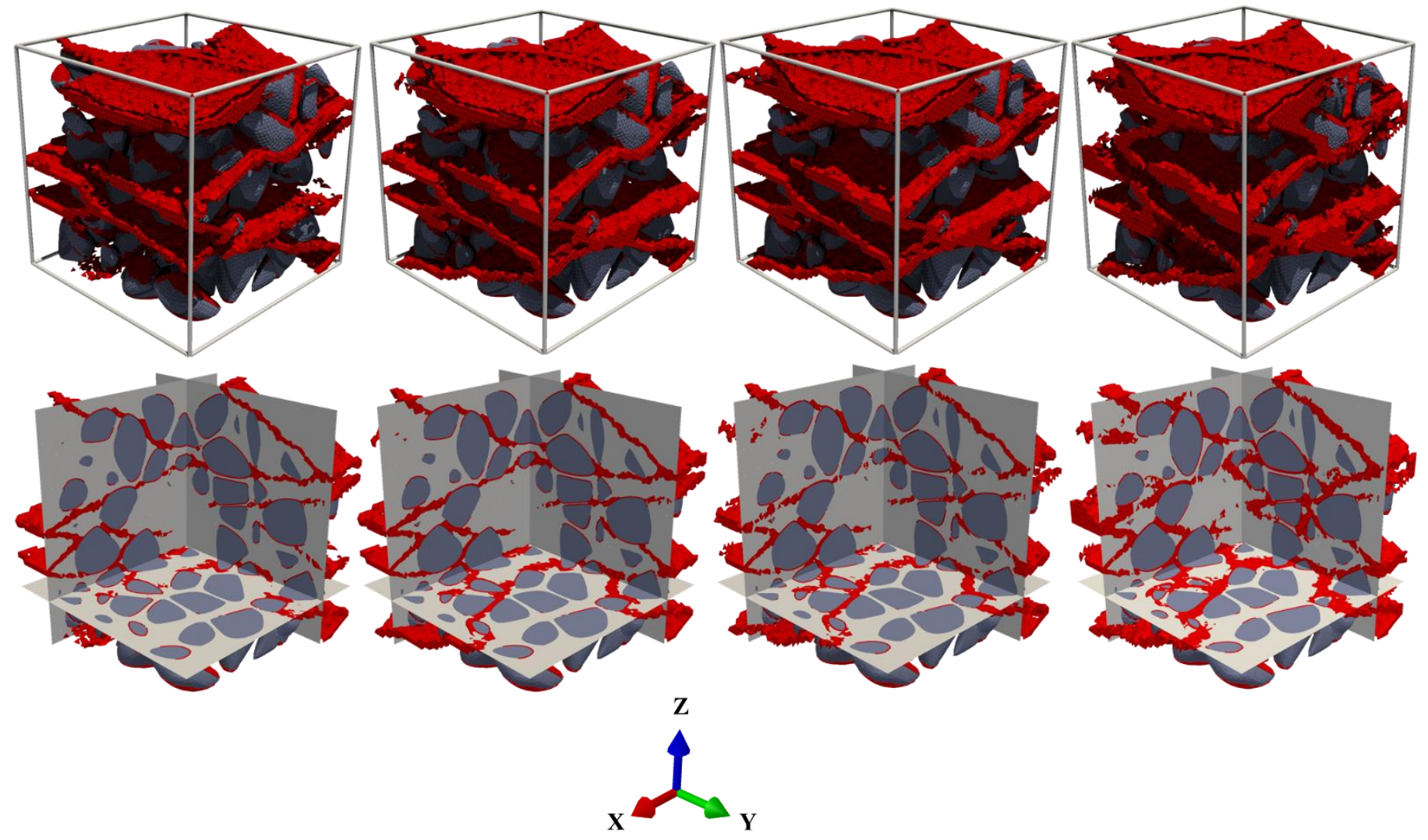

Fig. 18. Crack propagation in concrete under biaxial compression in $\mathrm{X}$ and $\mathrm{Y}$-directions where the damage is visible $(0.0015<\varepsilon \leq 0.004)$ at 4 stages for the model $\boldsymbol{G}$. The crack is visualized by deleting the corresponding elements (a) and the elements which SDEG values are greater than 0.9 (b and c). 

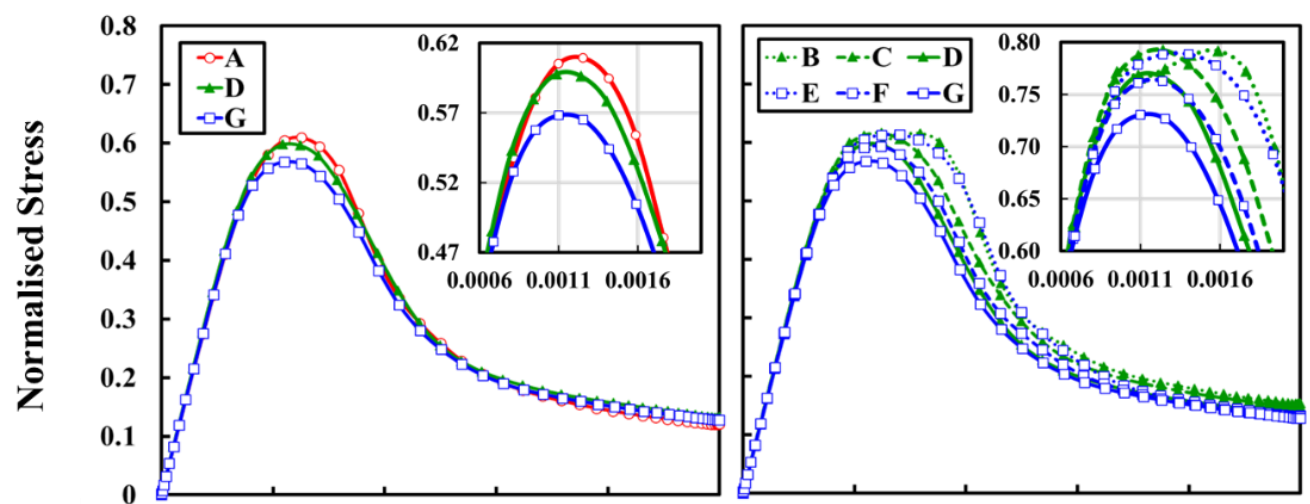

(a)
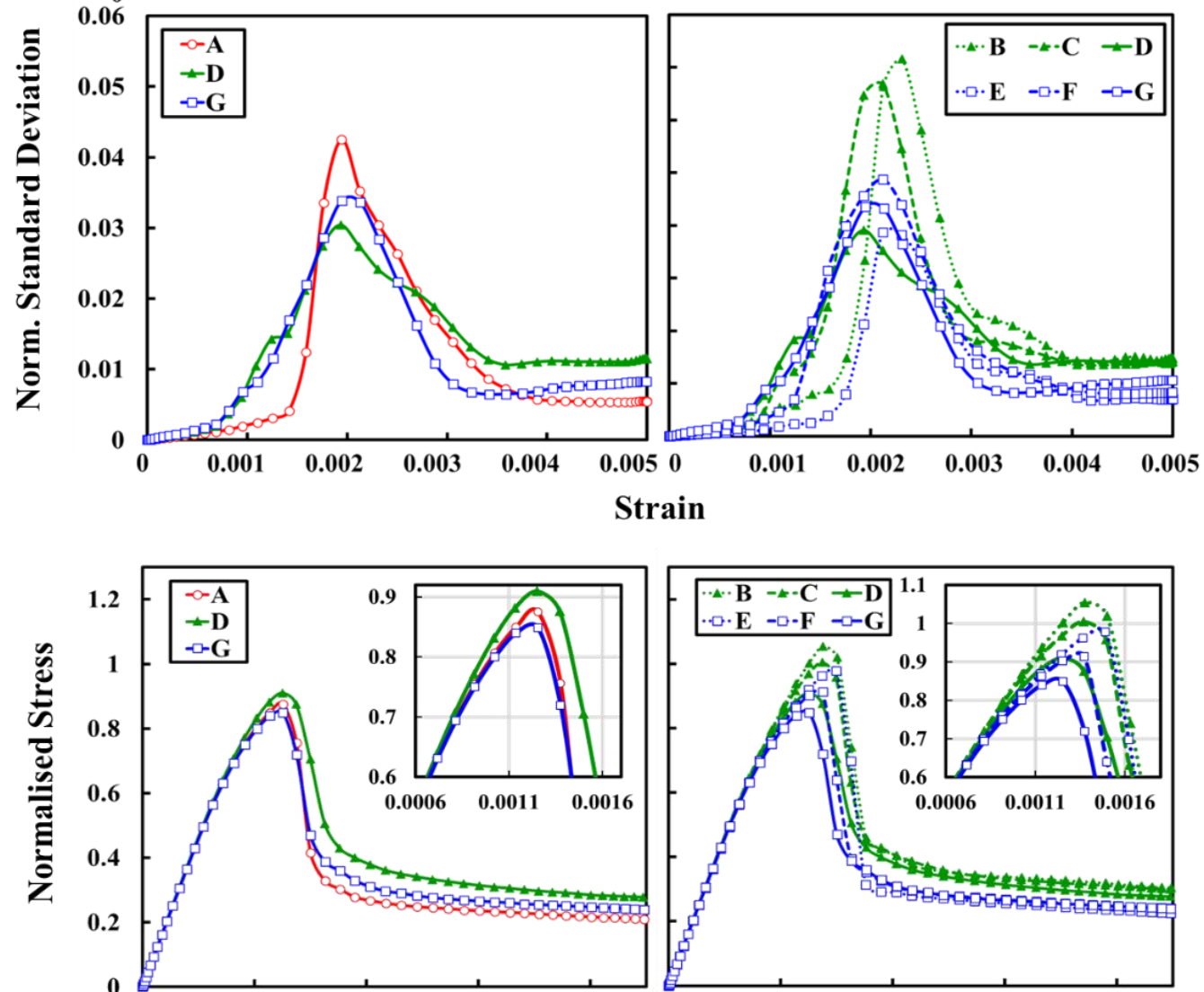

(b)

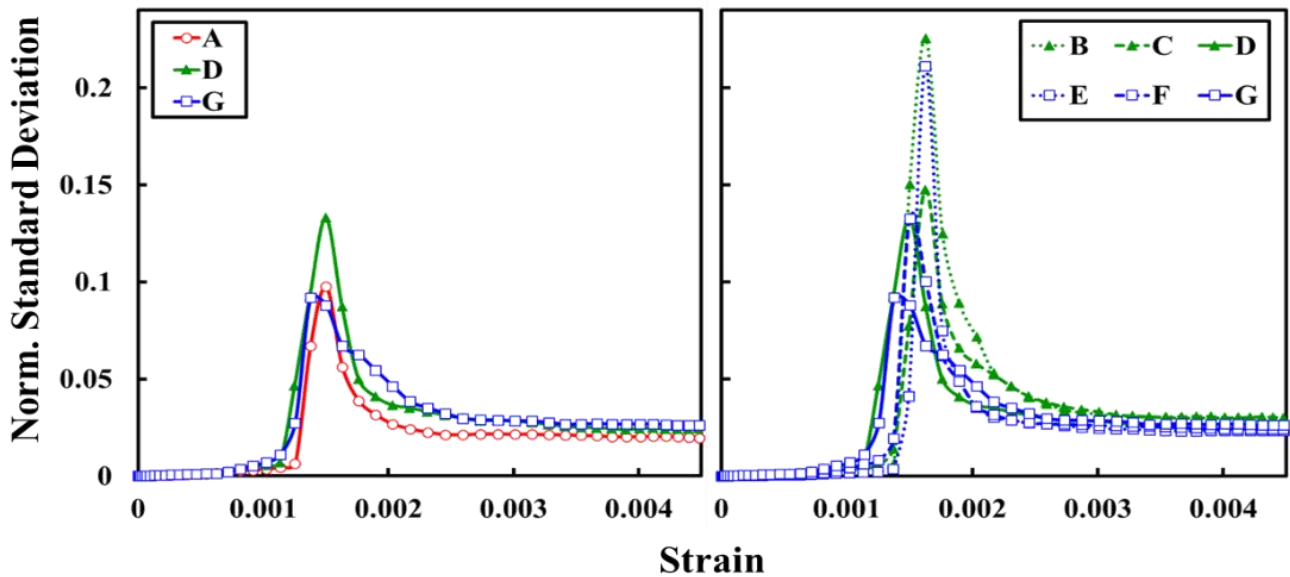

Fig. 19. Representative stress-strain and the corresponding standard deviation curves under uniaxial (a) and biaxial (b) loading. The stress values are normalised to the compressive strength of the mortar (45 MPa). The stress-strain curves around the peak stress are enlarged for better visualisation. 

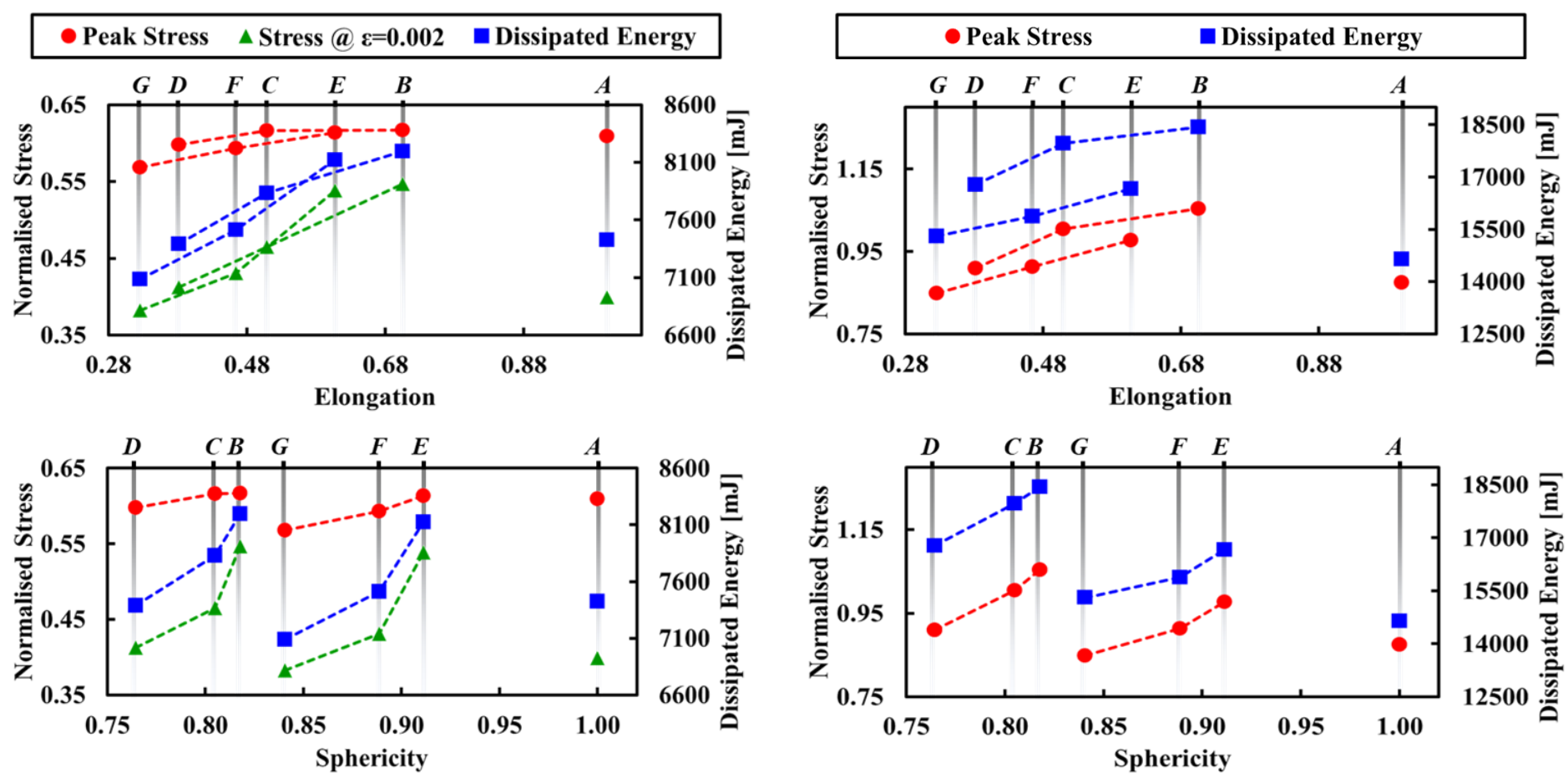

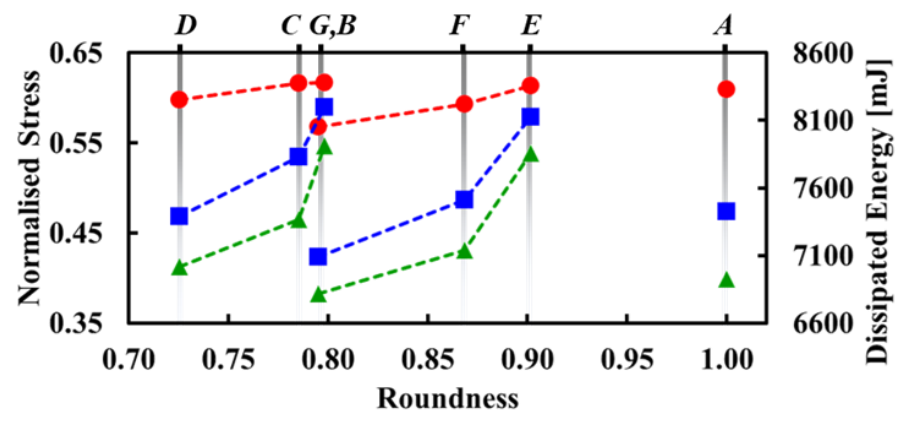

(a)

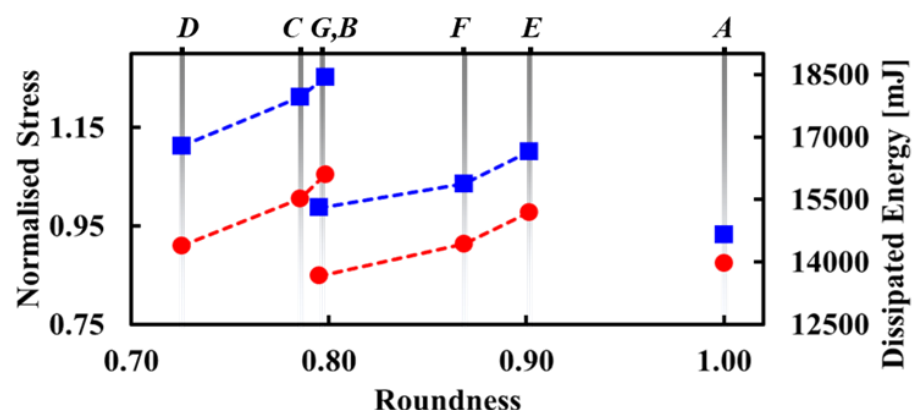

(b)

Fig. 20. Effects of the mean values of elongation ratio, sphericity and roundness on the critical values of stresses and the dissipated energy under uniaxial (a) and biaxial (b) loading. The data points are connected to guide the eye based on the concept summarized in Table 2. 
Table 1. The samples of different types of aggregate models are tabulated regarding the shape configurations including the irregularity $(r)$ and the iteration levels used in the Voronoi tessellation and the splining process respectively.

\begin{tabular}{|c|c|c|c|c|c|c|c|}
\hline $\mathrm{m}$ & Sphere & \multicolumn{3}{|c|}{ Polyhedral Voronoi Cell } & \multicolumn{3}{|c|}{ Splined-Voronoi Cell (Two Iteration Levels) } \\
\hline$r[$ Unit $]$ & - & 0 & 0.05 & 0.2 & 0 & 0.05 & 0.2 \\
\hline & $\boldsymbol{A}$ & $\boldsymbol{B}$ & $C$ & $D$ & $\boldsymbol{E}$ & $\boldsymbol{F}$ & $\boldsymbol{G}$ \\
\hline Models & & & & & & & \\
\hline
\end{tabular}

Table 2. The main concepts behind the design of the systems regarding the shape effects are outlined.

\begin{tabular}{ll}
\hline Comparison Set & Shape Effects \\
\hline$(\boldsymbol{A}, \boldsymbol{D}, \boldsymbol{G}$, Experiments $)$ & Geometric Type, Simplification \\
$(\boldsymbol{D}, \boldsymbol{G}) ;(\boldsymbol{C}, \boldsymbol{F}) ;(\boldsymbol{B}, \boldsymbol{E})$ & Splining Process \\
$(\boldsymbol{B}, \boldsymbol{C}, \boldsymbol{D})$ & Irregularity Level for Polyhedral Voronoi Cells \\
$(\boldsymbol{E}, \boldsymbol{F}, \boldsymbol{G})$ & Irregularity Level for Splined-Voronoi Cells \\
\hline
\end{tabular}

Table 3. Particle size distribution of coarse aggregates in concrete [13].

\begin{tabular}{lll}
\hline Sieve size $(\mathrm{mm})$ & Total percentage retained $(\%)$ & Total percentage passing $(\%)$ \\
\hline 12.7 & 0 & 100 \\
9.5 & 39 & 61 \\
4.75 & 90 & 10 \\
2.36 & 98.6 & 1.4 \\
\hline
\end{tabular}

Table 4. Mechanical properties used in the finite element simulations.

\begin{tabular}{llll}
\hline Material properties & Aggregate & Mortar & ITZ \\
\hline Young's modulus, $E(\mathrm{GPa})$ & 70 & 25 & 22 \\
Poisson's ratio, $v$ & 0.2 & 0.2 & 0.2 \\
Density, $\rho\left(\mathrm{kg} / \mathrm{m}^{3}\right)$ & 2600 & 2000 & 2000 \\
Tensile strength $(\mathrm{MPa})$ & - & 4 & 2 \\
Compressive strength $(\mathrm{MPa})$ & - & 45 & 22.5 \\
Fracture energy, $G_{f}(\mathrm{~N} / \mathrm{mm})$ & - & 0.06 & 0.03 \\
\hline
\end{tabular}

